\title{
Álgebras de Grupo Cujas Unidades Satisfazem uma Identidade de Grupo
}

\section{Raul Antonio Ferraz}

\author{
DISSERTAÇÃO APRESENTADA \\ $\mathrm{AO}$ \\ INSTITUTO DE MATEMÁTICA E ESTATÍSTICA \\ DA \\ UNIVERSIDADE DE SÃO PAULO \\ PARA \\ OBTENÇÃO DO GRAU \\ $\mathrm{DE}$ \\ MESTRE EM MATEMÁTICA \\ Área de Concentração: Álgebra \\ Orientador: Prof. Dr. Jairo Zacarias Gonçalves
}

Durante este trabalho, o autor teve o apoio financeiro do $\mathrm{CNPq}$ 


\section{Álgebras de Grupo \\ Cujas Unidades Satisfazem \\ uma Identidade de Grupo}

Este exemplar corresponde à redação final da dissertação devidamente corrigida e defendida por Raul Antonio Ferraz e aprovada pela comissão julgadora.

São Paulo, 26 de setembro de 1997.

Banca examinadora:

- Prof. Dr. Jairo Zacarias Gonçalves (Orientador) - IME - USP

- Prof. Dr. Francisco César Polcino Milies - IME - USP

- Prof. Dr. Guilherme Augusto de La Rocque Leal - UFRJ. 
Dedicada à memória de Mariana Issa Steiner (19/08/13-17/11/94) 
There is no permanent place in the world for ugly mathematics.

G. H. Hardy 


\section{Agradecimentos}

A Deus por me dar saúde, e me reconfortar nos momentos mais díficeis.

Ao meu orientador Prof. Dr. Jairo Zacarias Gonçalves pelo excelente trabalho de orientação e pelo apoio, incentivo, paciência e atenção constantes durante o trabalho.

Aos meus pais Thelmo e Neuza pelo carinho, amor, afeto, dedicação, atenção, ...enfim, por tudo que sempre fizeram por mim.

Aos meus familiares pelo apoio incentivo amor e carinho que sempre me dedicaram.

Ao Carlos Juiti Watanabe pela inestimável ajuda com os computadores das ET's., sem a qual esta tese não existiria. Gostaria de agradecer também ao Claus pelos vários conselhos sobre o Latex.

Aos meus colegas de graduação, mestrado, e doutorado pelo companheirismo.

A Luis Renato e Pedro pelas listas que tivemos a oportunidade de discutir juntos.

A Patrícia pela colaboração durante os exames de Cálculo e Funções Analíticas, sem a qual não conseguiria aprovação.

Ao Samuel pelas encomendas de livro que tive a oportunidade de fazer através de sua conta na Amazon.com.

A Daniel, Fernando e Walquíria pela companhia nos intermináveis lanches vespertinos.

Às professoras Profa. Dra. Iracema Bund, e Profa. Dra. Carmen Sílvia Cardassi pela oportunidade da Iniciação Científica que me despertou o gosto pela pesquisa em Matemática. Em particular à Profa. Dra. Carmen Sílvia Cardassi pelo carinho e atenção que sempre teve comigo durante o período em que me orientou.

A todos professores com os quais tive o prazer de ter assistido aulas e através delas enriquecer o meu conhecimento em Matemática. 
Aos professores da área de de Álgebra não comutativa, em especial as professoras Profa. Dra. Marly Mandia e Profa. Dra. Leilá Maria Vasconcelos Figueiredo.

Aos Professores Prof. Dr. Daniel Levcovitz e Prof. Dr. Paulo Agozzini Martins, pela atenção especial que tiveram por mim durante o mestrado.

Ao CNPq pelo apoio financeiro durante a elaboração desta dissertação. 


\section{Resumo:}

Seja $F[G]$ a álgebra de grupo do grupo $G$ sobre o corpo $F$, e seja $U(F[G])$ o seu grupo de unidades. O principal objetivo deste trabalho é investigar a validade da seguinte conjectura, devida a Brian Hartley.(problema 52, pag 307 de [Seh93]):

Conjectura: Se $G$ é um grupo de torção e $U(F[G])$ satisfaz uma identidade de grupo, então $F[G]$ satisfaz uma identidade polinomial.

Como suporte da afirmação acima provaremos:

Teorema 1:[GJV94],[GSV97]. A conjectura é verdadeira se $F$ é infinito.

Teorema 2:[Pas97]. Se $F$ é infinito, char $F=p>0$ e $G$ é um grupo de torção, então $U(F[G])$ satisfaz uma identidade de grupo se, e somente se, $G$ possui um subgrupo abeliano normal de índice finito, e $G^{\prime}$ é um p-grupo de expoente limitado.

\section{Abstract:}

Let $F[G]$ be the group ring of the group $G$ over the field $F$, and let $U(F[G])$ be its group of units. The main objective of this work is to investigate the following conjecture, due to Brian Hartley

Conjecture: If $G$ is a torsion group, and $U(F[G])$ satisfies a group identity, then $F[G]$ satisfy a polynomial identity.

In support of the statement above we prove:

Theorem 1: [GJV94],[GSV97] The conjecture is true when $F$ is infinite.

Theorem 2: [Pas97] If $F$ is infinite, $\operatorname{char} F=p>0$ and $G$ is a torsin group, then $U(F[G])$ satisfies a group identity if and only if, the group $G$ owns a $p$-abelian normal subgroup of finite index, and $G^{\prime}$ is a $p$-group of bounded expoent. 


\section{Índice de notações:}

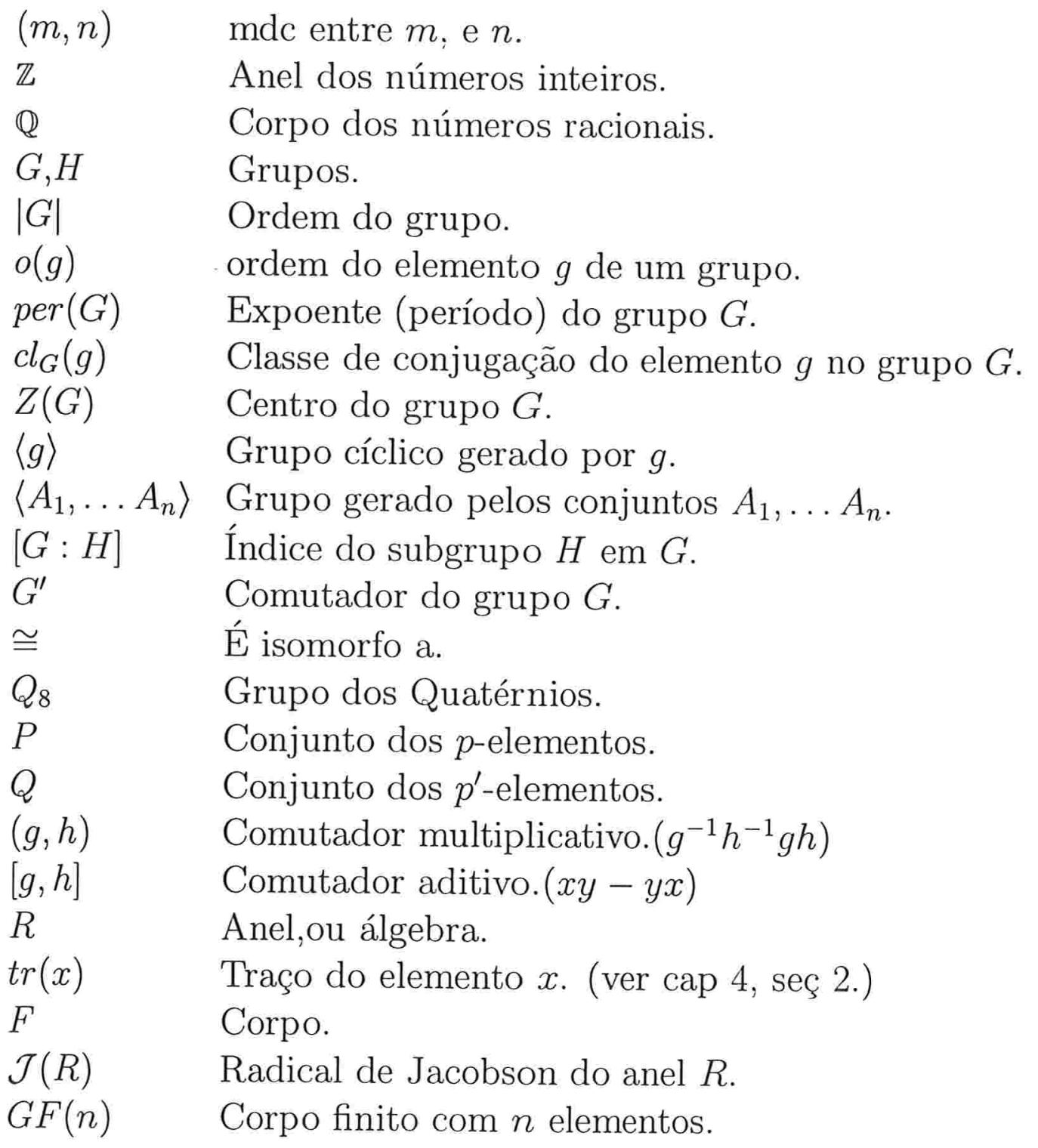




$\begin{array}{ll}M_{n}(R) & \text { Anel de matrizes } n \times n, \text { com entradas no anel } R . \\ {\left[\alpha_{i, j}\right]} & \text { Matriz pertencente a } M_{n}(R) \text {.ver proposição 4.14. } \\ Z(R) & \text { Centro do anel } R . \\ U(R) & \text { Grupo de unidades do anel } R . \\ \text { char }(R) & \text { Característica do anel. } \\ F[G] & \text { Anel de grupo do grupo } G, \text { sobre o corpo } F . \\ \Delta(G) & \text { Ideal de aumento do anel } F[G] . \\ \Delta(G, N), & \text { Kernel do epimorfismo canônico de } F[G] \text { em } F[G / \Lambda \\ \mathcal{F} & \text { Grupo livre. } \\ F\left\langle x_{1}, \ldots, x_{n}\right\rangle & \text { Álgebra livre sobre o corpo } F, \text { a } n \text { variáveis. } \\ \mathcal{A}, F\left\langle x_{1}, \ldots x_{n}\right\rangle[[t]] & \text { Série de potências de t em } F\left\langle x_{1}, \ldots x_{n}\right\rangle[[t]] . \\ \omega\left(x_{1}, \ldots x_{n}\right)=1 & \text { Identidade de grupo. } \\ p\left(x_{1}, \ldots x_{n}\right)=0 & \text { Identidade Polinomial. } \\ \Gamma_{n}\left(x_{1}, \ldots, x_{n}\right)=0 & \text { Identidade Polinomial Standard. } \\ S_{n} & \text { Grupo de permutações a } n \text { variáveis. } \\ \mathcal{S}(\sigma) & \text { Sinal da permutação sigma. } \\ \mathcal{H}(R) & \text { Conjunto dos elementos } a \in R, \text { tal que } a R \text { é } \\ & \text { nil de expoente limitado. } \\ \Phi(G) & \text { Grupo dos elementos de conjugação finita de } G . \\ \Phi_{p}(G) & \langle\Phi(G) \cap P\rangle \\ N(R) & \text { Soma dos ideais nilpotentes de } R . \\ \mathcal{N}(R) & \text { Conjunto dos elementos nilpotentes de } R . \\ H \leq G & \text { H é subgrupo de } G . \\ A \leq R & \text { A é subanel de } R . \\ A \rtimes H & \text { Produto semidireto de } A \text { por } H . \\ s u p p(h) & \text { Suporte do elemento } h . \\ & \end{array}$




\section{Conteúdo}

1 Introdução 1

1.1 Alguns Teoremas da Teoria de Grupos . . . . . . . . . . . . . . 2

1.2 Noções de Teoria de Anéis. . . . . . . . . . . . . . . . . . . . . 3

1.3 Anéis de grupo . . . . . . . . . . . . . . . . . . . 5

1.4 Identidades polinomiais e identidades de grupo. . . . . . . . . . . 7

2 Identidades no caso semiprimo 10

3 Caso Geral. 21

3.1 Introdução . . . . . . . . . . . . . . . . . . . . . . . . 21

$3.2 \quad$ O grupo $\Phi(G) \quad \ldots \ldots \ldots \ldots \ldots$

3.3 Relações entre o grupo $G$ e as identidades polinomiais satisfeitas por

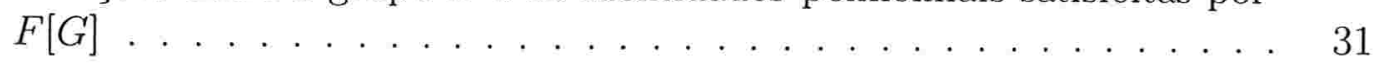

3.4 Demonstração do Teorema Principal . . . . . . . . . . . . . . . . . . 32

4 O caso modular-uma condição necessária e suficiente 41

4.1 Introdução: . . . . . . . . . . . . . . . . . . . . . . . . . 41

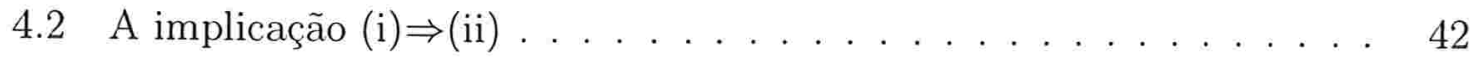

4.3 A implicação (ii) $\Longrightarrow$ (iii). . . . . . . . . . . . . . . . . 58

$\begin{array}{lc}\text { Bibliografia } & 63\end{array}$ 


\section{Capítulo 1}

\section{Introdução}

Seja $F[G]$ o anel de grupo do grupo $G$ sobre o corpo $F$, e seja $U(F[G])$ o seu grupo de unidades.

Investigando a estrutura de $U(F[G])$, Brian Hartley propôs:

Conjectura: Se a característica de $F$ é $p>0 G$ é um $p$-grupo localmente finito e $U(F[G])$ satisfaz uma identidade de grupo, então $F[G]$ satisfaz uma identidade polinomial. [War81]

Os primeiros resultados confirmando esta conjectura foram obtidos por Gonçalves e Mandel, [GM91], assumindo que o cardinal de $F$ é infinito e que a identidade satisfeita por $U(F[G])$ é uma identidade de semigrupo.

A seguir, em [GJV94], Giambruno, Jespers, e Valenti estudam o caso semiprimo para identidades de grupo. Posteriormente, em [GSV97], Giambruno, Sehgal, e Valenti conseguem remover esta restrição, e em [Pas97], Passman dá uma condição necessária e suficiente para que $U(F[G])$ satisfaça uma identidade de grupo quando $p>0$.

Muito recentemente, Chia Hsin Liu [Liu] demonstrou que se $G$ é um grupo de torção $F$ é um corpo qualquer (não necessariamente infinito), e $U(F[G]$ ) satisfaz uma identidade de grupo, então $F[G]$ satisfaz uma identidade polinomial, confirmando a Conjectura de Hartley.

Neste trabalho trataremos apenas do caso em que $F$ é infinito. Detalharemos a caracterização dos grupos, conforme Passman no caso em que $F$ tem característica $p>0$.

Inicialmente vamos enunciar alguns teoremas que nos serão úteis na compreensão desta dissertação. Demonstraremos alguns e indicaremos demonstrações detalhadas 
de outros.

No capítulo 2, estudaremos álgebras semiprimas, cujo o grupo de unidades satisfaz uma identidade de grupo. Concluindo, entre outros resultados, que nesta álgebra todo idempotente é central.

No capítulo 3, demonstraremos que $F[G]$ satisfaz uma identidade polinomial, sempre que $F$ é um corpo infinito, $G$ é um grupo de torção, e $U(F[G])$ satisfaz uma identidade de grupo.

Por fim, no capítulo 4, mostraremos que no caso em que $F$ tem característica $p>0, U(F[G])$ irá satisfazer uma identidade de grupo, se e somente se $G^{\prime}$ for $p$-grupo de período limitado, e $G$ possuir um subgrupo $A$ normal $p$-abeliano de índice finito.

\subsection{Alguns Teoremas da Teoria de Grupos}

Nesta seção enunciaremos alguns teoremas sobre teoria de grupos, muitos dos quais consagrados pelo constante uso em trabalhos nesta área.

Definição 1.1. Dizemos que um grupo $G$ é hamiltoniano se ele é da forma

$$
G=Q_{8} \times E \times A,
$$

onde $Q_{8}$ é grupo dos Quatérnios, E é um 2-grupo abeliano elementar e $A$ é um grupo abeliano de torção, no qual todo elemento tem ordem ímpar.

Teorema 1.2 (Dedekind-Baer). Seja $G$ um grupo. São equivalentes:

1. Todo subgrupo de $G$ é normal em $G$

2. Todo subgrupo cíclico de $G$ é normal em $G$

3. $G$ é ou abeliano ou hamiltoniano.

DemonstraÇÃo: pag 143, teorema 5.3.7 de [Rob95].

Notaremos por $G^{\prime}$ o subgrupo comutador de $G$, isto é,

$$
G^{\prime}=\left\langle(g, h)=g^{-1} h^{-1} g h \mid g, h \in G\right\rangle .
$$

Proposição 1.3. Seja $G$ um grupo com um subgrupo $H$ central de índice finito $[G$ : $H]=n$. Então $G^{\prime}$ é finito, com ordem menor ou igual a $\left(n^{2}\right)^{n^{3}}$. 
DemonstraÇÃo: pag 115, teorema 1.4 do capítulo 4 de [Pas77].

Definição 1.4. Seja $G$ um grupo. Dizemos que $G$ é localmente finito se todo subgrupo finitamente gerado de $G$ é finito.

Teorema 1.5 (Teorema de Schmidt). Seja G um grupo e $H$ um subgrupo normal de $G$. Se $H$ e $\frac{G}{H}$ são localmente finitos, então $G$ é localmente finito.

DEMONSTRAÇÃo: pag 429, teorema 14.3.1 de [Rob95].

Nem sempre é verdade que um subgrupo $H$ de um grupo $G$ finitamente gerado, é finitamente gerado. Se tomarmos $G$ o grupo livre de posto 2, e $H$ o seu comutador teremos que $G$ é finitamente gerado mas $H$ não. Porém, em um caso particular podemos concluir que $H$ é finitamente gerado.

Proposição 1.6. Seja $G$ um grupo finitamente gerado e $H$ um subgrupo de indice finito. Então $H$ é finitamente gerado.

DEMONSTRAÇÃO: pag 36, teorema 1.6.11 de [Rob95].

\subsection{Noções de Teoria de Anéis.}

Nesta seção vamos recordar alguns fatos básicos da teoria de anéis.

Definição 1.7. Seja $R$ um anel. Dizemos que $R$ é artiniano a esquerda se toda cadeia descendente de ideais a esquerda de $R$ é estacionária. Ou seja, se $I_{1}, I_{2}, \ldots$ , são ideais de R, com

$$
I_{1} \geq I_{2} \geq I_{3} \geq \ldots
$$

então existe um $k \in \mathbb{N}$ tal que para todo $n \geq k$

$$
I_{n}=I_{k} .
$$

Seja $R$ um anel unitário. Sabe-se que a intersecção dos ideais maximais a esquerda de $R$, coincide com a intersecção dos ideais maximais a direita. Usando tal fato definimos:

Definição 1.8. Seja $R$ um anel unitário. $O$ radical de Jacobson de $R$, como sendo a intersecção de todos ideais maximais a esquerda de $R$. E o denotamos por $\mathcal{J}(R)$, ou não havendo risco de dubiedade apenas por $\mathcal{J}$.

Proposição 1.9. Se $R$ é um anel artiniano e unitário, então $\mathcal{J}(R)$ é nilpotente. 
DemonstraÇÃo: ver pag 56 teorema 4.12 de [Lam91].

Definição 1.10. Dizemos que um anel $R$ é semiprimitivo se $\mathcal{J}(R)$ é o ideal nulo.

Definição 1.11. Dizemos que um anel $R$ é semiprimo se $R$ não tem ideais nilpotentes não triviais.

Sempre podemos encarar $R$ como sendo um módulo sobre si mesmo. Assim podemos definir anel semi-simples:

Definição 1.12. Seja $R$ um anel. Dizemos que $R$ é semi-simples a esquerda , se todo submódulo a esquerda $I$ de $R$ é somando direto de $R$. Isto é, para todo submódulo a esquerda de $I$, existe um submódulo a esquerda $J$, tal que $I \cap J=(0)$, $e I+J=R$. Denotamos a soma direta de $I$ e $J$ por,

$$
I \oplus J
$$

É fato conhecido que um anel unitário é semi-simples a esquerda se e somente se é semi simples a direita. Portanto usaremos o termo semi-simples.

Teorema 1.13. Seja $R$ um anel unitário artiniano a esquerda. São equivalentes:

1. $R$ é semiprimitivo;

2. $R$ é semiprimo;

3. $R$ é semi-simples.

DEMONSTRAÇÃo: pag 184 teorema 11.7 de [Lam91]

Teorema 1.14 (Wedderburn-Artin). Seja $R$ um anel unitário semisimples. Então $R \cong \bigoplus_{i=1}^{r} M_{n_{i}}\left(D_{i}\right)$, onde cada $D_{i}$ é um anel com divisão, $r$ é único, e os pares $\left(D_{i}, n_{i}\right)$, são únicos a menos de permutação.

DEMONSTRAÇÃO: pag 35 teorema 3.5 [Lam91]

Teorema 1.15 (Cayley-Hamilton). Seja $C$ um anel comutativo, não necessariamente unitário. Seja A uma matriz pertencente a $M_{n}(C)$. Se $p_{A}$ é o polinômio característico de $A$ (isto é $p_{A}(\lambda)=\operatorname{det}(\lambda I-A)$ ), então $p_{A}(A)=0$.

Demonstração: pag 18 teo 1.3.18 de [Row80].

Para encerrar esta seção um teorema que não é básico, mas nos será muito útil futuramente. 
Teorema 1.16 (Gonçalves). Seja $D$ um anel com divisão não comutativo, que tenha dimensão finita sobre seu centro $Z$. Então $D^{*}=D-0$ contém um grupo livre de posto 2.

DEMONSTRAÇÃo: ver [JZG84].

\subsection{Anéis de grupo}

Vamos nesta seção introduzir a noção de Anel de Grupo, e estudar algumas de suas propriedades.

Definição 1.17. Seja $A$ um anel e $G$ um grupo. O anel de grupo do grupo $G$ sobre o anel $A$ notado por $R=A[G]$, é o conjunto de todas somas finitas formais:

$$
\sum_{g \in G} a_{g} g
$$

com $g \in G$ e $a_{g} \in A$, e com a seguinte estrutura de anel. A soma é definida como

$$
\sum_{g \in G} a_{g} g+\sum_{g \in G} b_{g} g=\sum_{g \in G}\left(a_{g}+b_{g}\right) g
$$

e o produto

$$
\sum_{g \in G} a_{g} g \cdot \sum_{h \in G} b_{h} h=\sum_{g, h \in G}\left(a_{g} b_{h}\right) g h .
$$

Também tem a estrutura de A-módulo:

$$
a \cdot \sum_{g \in G} a_{g} g=\sum_{g \in G} a \cdot a_{g} g
$$

Podemos notar que se o anel $A$ é unitário, o anel $A[G]$ também o será, tendo como unidade o elemento $1_{A} 1_{G}$.

Vamos centrar nossas atenções para o caso específico em o anel $A$ é um corpo. Usaremos a letra $F$ para denotar tal corpo.

Vamos estudar alguns ideais de $F[G]$.

Seja $H$ subgrupo de $G$. Notaremos por $\pi_{H}$ a função:

$$
\begin{aligned}
\pi_{H}: F[G] & \longrightarrow F[H] \\
\sum_{g \in G} \alpha_{g} g & \longrightarrow \sum_{g \in H} \alpha_{g} g
\end{aligned}
$$

Ou seja tiramos da soma os elementos que não estão em $H$. 
Lema 1.18. Sejam $F$ um corpo, $G$ um grupo, $H$ um subgrupo de $G$, e $Y$ uma transversal a direita de $H$ em $G$. Então para todo elemento $\alpha \in F[G]$, existem e são únicos $\alpha_{y} \in F[H]$ tal que:

$$
\alpha=\sum_{y \in Y} \alpha_{y} y
$$

Além disso temos $\alpha_{y}=\pi_{H}\left(\alpha y^{-1}\right) \in K[G]$

DEMONSTRAÇÃo: ver pagina 6, lema 1.3 do capítulo 1 de [Pas77].

Definição 1.19. Dizemos que um ideal $I$ de $F[H]$, é $G$-invariante, se para todo $g$ pertencente a $G$ temos $g^{-1} I g \leq I$.

Lema 1.20. Seja $G$ um grupo e $F$ um corpo, $H \triangleleft G$, e seja $\mathcal{I}$ um ideal de $F[G]$. São equivalentes:

1. $\mathcal{I}=I \cdot F[G]$, para algum $I$ ideal $G$-invariante de $F[H]$;

2. $\mathcal{I}=(\mathcal{I} \cap F[H]) \cdot F[G]$;

3. $\mathcal{I}=\pi_{H}(\mathcal{I}) \cdot F[G]$.

Nestas condições temos:

$$
I=\mathcal{I} \cap F[H]=\pi_{H}(\mathcal{I})
$$

DemonstraÇÃo: ver pag 8, lema 1.6 do capítulo 1 de [Pas77].

Vamos introduzir, agora, a noção de Ideal de Aumento, que nos será muito útil posteriormente.

Definição 1.21. Seja $F[G]$ um anel de grupo, e seja a aplicação $\pi$ :

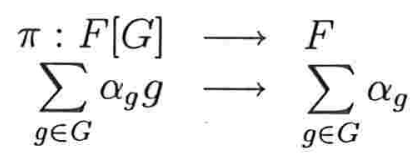

$\pi$ assim definido é homomorfismo de anéis. Tal homomorfismo é denominado a aplicação de aumento de $F[G]$. $O$ ideal de aumento de $F[G]$, é o kernel desta aplicação, e o notamos por $\Delta(G)$ 
Definição 1.22. Seja $N \triangleleft G$ e seja $\pi^{\prime}$ o homomorfismo:

$$
\begin{aligned}
\pi^{\prime}: F[G] & \longrightarrow F\left[\frac{G}{N}\right] \\
\sum_{g \in G} \alpha_{g} g & \longrightarrow \sum_{g \in G} \alpha_{g} g N
\end{aligned}
$$

Notamos por $\Delta(G, N)$, o kernel de $\pi^{\prime}$. O epimorfismo $\pi^{\prime}$ será denominado epimorfismo canônico de $F[G]$ em $F\left[\frac{G}{N}\right]$.

Nas condições acima, vale a igualdade:

$$
F[G](\Delta(N))^{n}=(\Delta(G, N))^{n}=(\Delta(N))^{n} F[G]
$$

Para maiores detalhes ver o lema 1.8 do capítulo 1 de [Pas77].

Proposição 1.23. Sejam $G$ um grupo não trivial e $F$ um corpo. Então $\Delta(G)$ é nilpotente se e somente se $\operatorname{car}(F)=p>0$ e $G$ for um p-grupo finito. Se a característica de $F$ for 0 , então $\Delta(G)$ é não nilpotente.

DemonstraçÃo: ver pag 70 teorema 1.6 do capítulo 3 do [Pas77].

Da proposição acima e da observação anterior segue o corolário:

Corolário 1.24. Sejam $G$ um grupo, $N$ um p-subgrupo finito, normal em $G$ e $F$ um corpo de característica $p>0$. Então $\Delta(G, N)$ é nilpotente.

\subsection{Identidades polinomiais e identidades de gru- po.}

Definição 1.25. Seja $\omega\left(x_{1}, \ldots x_{n}\right) \neq 1$ um elemento do grupo livre de posto $n$. Dizemos que $\omega=1$ é uma identidade de grupo para $G$, se para todos $g_{1}, g_{2}, \ldots g_{n} \in G$ temos

$$
\omega\left(g_{1}, \ldots, g_{n}\right)=1
$$

Sejam $R$ e $S$ dois anéis unitários, consideraremos como homomorfismos de $R$ em $S$, apenas os homomorfismos que levem $1_{R}$ em $1_{S}$. Com esta consideração vamos enunciar

Proposição 1.26. Sejam $R$ e $S$ anéis unitários, e seja $f$ um epimorfismo de $R$ em $S$. Se o kernel de $f$ é nilpotente, e $U(R)$ satisfaz a identidade de grupo $\omega\left(x_{1}, \ldots, x_{n}\right)=1$, então $U(S)$ também satisfaz $\omega=1$. 


\section{Demonstração:}

Como $f$ é epimorfismo temos que para todo $a \in S$, existe um $a_{1} \in R$, tal que $f\left(a_{1}\right)=a$. Inicialmente demonstraremos que se $a=f\left(a_{1}\right) \in U(S)$, então $a_{1} \in U(R)$ :

Seja $a \in U(S)$, existe $b=f\left(b_{1}\right) \in U(S)$ tal que

$$
a b=b a=1_{S}
$$

Assim,

$$
f\left(a_{1} b_{1}\right)=f\left(a_{1}\right) f\left(b_{1}\right)=a b=1_{S}=f\left(1_{R}\right),
$$

$\operatorname{logo} 1_{R}-a_{1} b_{1}$ pertence ao kernel de $f$.

Seja $j=1_{R}-a_{1} b_{1}$, como kernel de $f$ é nilpotente, temos que existe $n$, tal que $j^{n}=0$.

Sendo assim temos:

$$
\left(1_{R}-j\right)\left(1_{R}+j+\cdots+j^{n-1}\right)=1_{R}-j^{n}=1_{R},
$$

e portanto

$$
\left(a_{1} b_{1}\right)\left(1_{R}+j+\cdots+j^{n-1}\right)=1_{R} .
$$

Concluimos, portanto, que $a_{1}$ é inversível a direita.

De forma análoga podemos concluir que $a_{1}$ é inversível a esquerda, e que portanto $a_{1} \in U(R)$. Vamos agora demonstrar que $U(S)$ satisfaz $\omega=1_{S}$ :

Sejam $s_{1}, \ldots, s_{n} \in U(S)$, e digamos $s_{i}=f\left(r_{i}\right) \operatorname{com} r_{i} \in U(R)$.

Como $U(R)$ satisfaz $\omega=1_{R}$, teremos:

$$
\omega\left(s_{1}, \ldots s_{n}\right)=\omega\left(f\left(r_{1}\right), \ldots, f\left(r_{n}\right)\right)=f\left(\omega\left(r_{1}, \ldots, r_{n}\right)\right)=f\left(1_{R}\right)=1_{S} .
$$

Para todos $s_{1}, \ldots s_{n} \in S$.

Um caso especial da proposição acima, que utilizaremos a posteriori é:

Proposição 1.27. Sejam F um corpo de característica $p>0, G$ um grupo, e $N$ um p-subgrupo finito normal em $G$. Se $U(F[G])$ satisfaz a identidade de grupo $\omega=1$ então $U\left(F\left[\frac{G}{N}\right]\right)$ também irá satisfazer $\omega=1$.

\section{DEMONSTRAÇÃO:}

Seja $\pi$ o epimorfismo canônico de $F[G]$ em $F\left[\frac{G}{N}\right]$.

Pelo corolário 1.24, o kernel de $\pi$ é nilpotente.

Segue da proposição anterior que $U\left(F\left[\frac{G}{N}\right]\right)$ satisfaz $\omega=1$. 
Definição 1.28. Seja $F\left\langle x_{1}, x_{2}, \ldots\right\rangle$ a àlgebra livre de posto enumerável sobre o corpo $F$. Dizemos que uma $F$-álgebra $R$ satisfaz uma identidade polinomial de grau $s$ se existe $p\left(x_{1}, x_{2}, \ldots, x_{n}\right) \in F\left\langle x_{1}, x_{2}, \ldots\right\rangle, p \neq 0$ com grau de $p$ igual a $s$ tal que

$$
p\left(r_{1}, r_{2}, \ldots, r_{n}\right)=0,
$$

para todos $r_{1}, r_{2}, \ldots r_{n} \in R$.

Teorema 1.29 (Kaplansky). Seja $R$ uma álgebra sobre um corpo F. Se $R$ satisfaz uma identidade polinomial de grau $n$, então $R$ satisfaz uma identidade polinomial da forma

$$
f\left(x_{1}, x_{2}, \ldots x_{n}\right)=\sum_{\sigma \in S_{n}} a_{\sigma} x_{\sigma(1)} \cdots x_{\sigma(n)}=0
$$

com os $a_{\sigma}$ pertencentes a $F$, não todos nulos.

DemonstraÇÃo: ver pag 170, Lema 1.1 do capítulo 5 de [Pas77].

Definição 1.30. Dizemos que um anel $R$ satisfaz a identidade polinomial standard de grau $n$, se para todos $r_{1}, \ldots, r_{n}$ vale:

$$
\Gamma_{n}\left(r_{1}, \ldots, r_{n}\right)=\sum_{\sigma \in S_{n}} \mathcal{S}(\sigma) r_{\sigma(1)} \cdots r_{\sigma(n)}=0
$$

onde $\mathcal{S}(\sigma)$ é a assinatura (ou o sinal) da permutação $\sigma$.

Teorema 1.31 (Amitsur-Levitsky). Seja $R$ um anel unitário comutativo. Então $M_{n}(R)$ satisfaz a identidade polinomial standard de grau $2 n$

DemonstraÇÃo: ver pag 175, teorema 1.9 do capítulo 5 de [Pas77].

Definição 1.32. Seja $R$ uma álgebra sobre $F$. Dizemos que $f$ é um polinômio multilinear generalizado se $f$ é da forma:

$$
f\left(\zeta_{1}, \ldots \zeta_{n}\right)=\sum_{\sigma \in S_{n}} f^{\sigma}\left(\zeta_{1} \ldots \zeta_{n}\right),
$$

onde $f^{\sigma}$ é um polinômio da forma,

$$
f^{\sigma}\left(\zeta_{1}, \ldots \zeta_{n}\right)=\sum_{j=1}^{a_{\sigma}} \alpha_{0, \sigma, j} \zeta_{1} \cdots \alpha_{n-1, \sigma, j} \zeta_{n} \alpha_{n, \sigma, j},
$$

onde cada $\alpha_{i, \sigma, j}$ é um elemento de $R$.

Definição 1.33. Dizemos que $f$ é um polinômio multilinear generalizado não degenerado, se para algum $\sigma, f^{\sigma}\left(\zeta_{1}, \ldots \zeta_{n}\right)$, não é identidade polinomial generalizada para a álgebra $R$, ou seja existem $r_{1}, \ldots r_{n} \in R$, tal que $f^{\sigma}\left(r_{1}, \ldots r_{n}\right) \neq 0$. 


\section{Capítulo 2}

\section{Identidades no caso semiprimo}

Neste capítulo estudaremos a estrutura de um anel semiprimo $A$ cujo grupo de unidades $U(A)$ satisfaz uma identidade de grupo. Concluiremos, dentre outros resultados, que se $A$ é uma álgebra semiprima sobre um domínio de integridade e o grupo de unidades de $A$ satisfaz uma identidade de grupo, todos os idempotentes de $R^{-1} A$ são centrais.

Vamos começar com uma proposição típica da teoria combinatória de grupos:

Proposição 2.1. Seja $G$ um grupo. Se G satisfaz uma identidade de grupo

$$
\omega\left(x_{1}, \ldots, x_{n}\right)=1,
$$

então $G$ satisfaz uma identidade em duas variaveis $\nu\left(y_{1}, y_{2}\right)=1$.

DemonstraçÃo:

Seja

$$
\omega\left(x_{1}, \ldots, x_{n}\right)=x_{i_{1}}^{r_{1}} \cdots x_{i_{k}}^{r_{k}}
$$

com $i_{j} \neq i_{j+1}, i_{j} \in\{1, \ldots, n\}$ Tomemos a identidade de grupo $\nu$ :

$$
\nu\left(y_{1}, y_{2}\right)=y_{1}^{-i_{1}} y_{2}^{r_{1}} y_{1}^{i_{1}-i_{2}} \cdots y_{1}^{i_{k-1}-i_{k}} y_{2}^{r_{k}} y_{1}^{i_{k}}
$$

Como $i_{j}-i_{j+1} \neq 0$ para todo $j$, temos que $\nu$ é não trivial.

Vamos mostrar que $G$ satisfaz $\nu=1$.

Sejam $g_{1}, g_{2} \in G$ Vamos definir $n h_{i}^{\prime}$ s como abaixo:

$$
h_{i}=g_{1}^{-i} g_{2} g_{1}^{i}
$$


Assim temos para todo $r$ inteiro

$$
\left(h_{i}\right)^{r}=g_{1}^{-i} g_{2}^{r} g_{1}^{i}
$$

Por um lado, usando nossa hipótese, temos:

$$
\omega\left(h_{1}, \ldots, h_{n}\right)=1
$$

E por outro lado:

$$
\begin{gathered}
\omega\left(h_{1}, \ldots, h_{n}\right)=h_{i_{1}}^{r_{1}} \cdots h_{i_{k}}^{r_{k}}= \\
g_{1}^{-i_{1}} g_{2}^{r_{1}} g_{1}^{i_{1}} g_{1}^{-i_{2}} \cdots g_{2}^{r_{k}} g_{1}^{i_{k}}= \\
g_{1}^{-i_{1}} g_{2}^{r_{1}} g_{1}^{i_{1}-i_{2}} \cdots g_{2}^{r_{k}} g_{1}^{i_{k}}=\nu\left(g_{1}, g_{2}\right)
\end{gathered}
$$

Portanto concluimos que $\nu\left(g_{1}, g_{2}\right)=1$ para todos $g_{1}, g_{2} \in G$. Logo $G$ satisfaz $\nu=1$.

O próximo teorema tomará parte central, na demonstração do principal teorema deste capítulo, e na demonstração de um dos lados do teorema do último capítulo. Vamos a ele:

Teorema 2.2 (Giambruno, Jespers e, Valenti). Seja A uma álgebra unitária sobre um dominio comutativo infinito $R$, cujo grupo de unidades $U(A)$ satisfaz uma identidade de grupo. Então existe um inteiro positivo $k$ tal que, se $a, b, c \in A e$ $a^{2}=b c=0$ então bacA é um ideal nil a direita de expoente limitado menor ou igual $a k$.

\section{DEMONSTRAÇÃO:}

Inicialmente vamos provar o caso em que $b=c$ :

Para tanto vamos mostrar que se existem $c, d \in A$ tal que $c^{2}=d^{2}=0$, então $d c$ é nilpotente, sendo que o grau de nilpotência de $d c$ só depende da natureza da identidade de grupo satisfeita por $U(A)$, e não da escolha de $c$ e $d$.

Usando a Proposição 2.1, podemos supor que $U(A)$ satisfaz uma identidade de grupo em duas variaveis do tipo:

$$
\omega\left(y_{1}, y_{2}\right)=y_{1}^{r_{1}} y_{2}^{r_{2}} y_{1}^{r_{3}} \cdots y_{1}^{r_{2 k+1}}=1
$$

Além disso, se a identidade vale para todo $x \in G$, vale também para $x^{-1}$, e portanto podemos supor $r_{1}>0$. 
Sejam $c, d \in A$ tal que $c^{2}=d^{2}=0$, e seja $\lambda \in R$. Façamos:

$$
\begin{gathered}
x_{1}=1+\lambda d c d \\
x_{2}=1+\lambda c \\
x_{3}=1+\lambda(1-d)(c d c)(1+d) .
\end{gathered}
$$

Vale observar que $x_{1}, x_{2}, x_{3} \in U(A)$

$$
\begin{gathered}
x_{1} \cdot(1-\lambda d c d)=1-\lambda^{2} d c \underbrace{d^{2}}_{=0} c=1 \\
x_{2} \cdot(1-\lambda c)=1-\lambda^{2} \underbrace{c^{2}}_{=0}=1
\end{gathered}
$$

$x_{3} \cdot(1-\lambda(1-d)(c d c)(1+d))=1-\lambda^{2}(1-d)(c d c) \underbrace{(1+d)(1-d)}_{=1}(c d c)(1+d)=$

$$
1-\lambda^{2}(1-d) c d \underbrace{c^{2}}_{=0} d c(1+d)=1
$$

E portanto $x_{1} x_{2}$ e $x_{1} x_{3}$ também são unidades de $A$. Assim:

$$
\left(x_{1} x_{2}\right)^{r_{1}}\left(x_{1} x_{3}\right)^{r_{2}} \cdots\left(x_{1} x_{2}\right)^{r_{2 k+1}}=1
$$

Podemos notar que $\left(x_{1} x_{2}\right)^{r_{1}}\left(x_{1} x_{3}\right)^{r_{2}} \cdots\left(x_{1} x_{2}\right)^{r_{2 k+1}}$ pode ser escrito na forma $\prod_{i=1}^{\epsilon} x_{j_{i}} \delta_{i}$, onde $\epsilon \leq 2\left(\left|r_{1}\right|+\cdots+\left|r_{2 k+1}\right|\right), \delta_{i}$ é 1 ou -1 , e $x_{j_{i}} \neq x_{j_{i+1}}\left(j_{i} \in\{1,2,3\}\right.$ para todo $\left.i\right)$. Além disso $\epsilon \geq 2\left(\left|r_{1}\right|+\cdots+\left|r_{2 k+1}\right|\right)-4 k$, visto que existem no máximo $2 k$ encontros de $x_{1}{ }^{-1}$ com $x_{1}$ e que em cada encontro temos 2 elementos a menos. Em particular temos $\epsilon \geq 2$.

Vamos analisar agora $\omega\left(x_{1} x_{2}, x_{1} x_{3}\right)$ como função de $c$ e $d$ e $\lambda$ :

\section{- Afirmação:}

Para todo $\lambda \in R$, podemos escrever $\omega\left(x_{1} x_{2}, x_{1} x_{3}\right)$ como

$$
\prod_{i=1}^{\epsilon} x_{j_{i}}^{\delta_{i}}=1+\sum_{i=1}^{\epsilon} \lambda^{i} p_{i}(c, d)
$$

onde os $p_{i}$ 's são polinômios na álgebra livre em duas variáveis sobre o anel $R, R\langle x, y\rangle$, que não dependem da escolha de $\lambda$, e $p_{\epsilon}(c, d)= \pm l_{\epsilon}$, com $l_{\epsilon}$ da forma:

$$
l_{\epsilon}=\left\{\begin{array}{ccc}
(d c)^{\alpha} d & \text { se } & j_{\epsilon}=1 \\
(d c)^{\alpha} & \text { se } & j_{\epsilon}=2 \\
(d c)^{\alpha}(1+d) & \text { se } & j_{\epsilon}=3
\end{array}\right.
$$


Onde $\alpha$ é um inteiro positivo menor ou igual a $2 \epsilon$.

DEMONSTRAÇÃO:

Seja

$$
\omega\left(x_{1} x_{2}, x_{1} x_{3}\right)=\prod_{i=1}^{\epsilon} x_{j_{i}}^{\delta_{i}}
$$

Vamos mostrar que a afirmação é verdadeira por indução em $\epsilon$.

Começaremos a indução $\operatorname{com} \epsilon=2$, visto que $\epsilon \geq 2$ independente de $\omega$.

- $\epsilon=2$ :

Como supomos $r_{1}>0$, temos

$$
\begin{gathered}
\omega=x_{1} x_{2}=(1+\lambda d c d)(1+\lambda c)= \\
1+\lambda d c d+\lambda c+\lambda^{2} d c d c=1+\lambda(d c d+c)+\lambda^{2}(d c)^{2}
\end{gathered}
$$

Logo $p_{\epsilon}(c, d)=p_{2}(c, d)=(d c)^{2}$, e portanto a afirmação vale se $\epsilon=2$.

- $\epsilon>2$ : Vamos supor que a afirmação valha para $\epsilon-1$ e vamos provar que vale para $\epsilon$ :

Usaremos $\alpha^{\prime}$ e $p_{i}^{\prime}$ para descrevermos o caso $\epsilon-1$. Por convenção $p_{0}(c, d)=1$. Consideraremos várias possibilidades, utilizando a hipótese que $x_{j-1} \neq x_{j}$.

(1) $j_{\epsilon-1}=1, \quad j_{\epsilon}=2$

$$
\begin{gathered}
\prod_{i=1}^{\epsilon} x_{j_{i}}^{\delta_{i}}=\underbrace{\left(1+\sum_{i=1}^{\epsilon-2} \lambda^{i} p_{i}^{\prime}(c, d) \pm \lambda^{\epsilon-1} \cdot(d c)^{\alpha^{\prime}} d\right)}_{\prod_{i=1}^{\epsilon-1} x_{j_{i}}^{\delta_{i}}} \underbrace{\left(1+\delta_{\epsilon} \lambda c\right)}_{x_{j_{\epsilon}}^{\delta_{\epsilon}}}= \\
1+\lambda \delta_{\epsilon} c+\sum_{i=1}^{\epsilon-2} \lambda^{i} p_{i}^{\prime}(c, d)+\sum_{i=1}^{\epsilon-2} \delta_{\epsilon} \lambda^{i+1} p_{i}^{\prime}(c, d) c+ \\
\pm \lambda^{\epsilon-1}(d c)^{\alpha^{\prime}} d \pm \delta_{\epsilon} \lambda^{\epsilon}(d c)^{\alpha^{\prime}} d c= \\
1+\sum_{i=1}^{\epsilon-1} \lambda^{i} \underbrace{\left(\delta_{\epsilon} p_{i-1}^{\prime}(c, d) c+p_{i}^{\prime}(c, d)\right)}_{p_{i}(c, d)} \pm \lambda^{\epsilon}(d c)^{\alpha^{\prime}+1}
\end{gathered}
$$

Como $\alpha^{\prime} \leq 2(\epsilon-1)$, então $\alpha=\alpha^{\prime}+1 \leq 2 \epsilon$ e portanto a afirmação é verdadeira. 
(2) $j_{\epsilon-1}=1, \quad j_{\epsilon}=3$

$$
\begin{gathered}
\prod_{i=1}^{\epsilon} x_{j_{i}}^{\delta_{i}}=\underbrace{\left(1+\cdots \pm \lambda^{\epsilon-1} x_{j_{i}}^{\delta_{i}}\right.}_{\prod_{j_{\epsilon}}^{\delta_{\epsilon}}} \cdot(d c)^{\alpha^{\prime}} d) \underbrace{\left(1+\delta_{\epsilon} \lambda(1-d)(c d c)(1+d)\right)}= \\
1+\cdots \pm \lambda^{\epsilon}(d c)^{\alpha^{\prime}} d(1-d)(c d c)(1+d)= \\
1+\cdots \pm \lambda^{\epsilon}(d c)^{\alpha^{\prime}+2}(1+d)
\end{gathered}
$$

Como $\alpha^{\prime} \leq 2(\epsilon-1)$, então $\alpha=\alpha^{\prime}+2 \leq 2 \epsilon$ e portanto a afirmação é verdadeira.

(3) $j_{\epsilon-1}=2, \quad j_{\epsilon}=1$

$$
\begin{gathered}
\prod_{i=1}^{\epsilon} x_{j_{i}}^{\delta_{i}}=\underbrace{\left(1+\cdots \pm \lambda^{\epsilon-1} \cdot(d c)^{\alpha^{\prime}}\right)}_{\prod_{i=1}^{\epsilon-1} x_{j_{i}}^{\delta_{i}}} \underbrace{\left(1+\delta_{\epsilon} \lambda d c d\right)}_{x_{j_{\epsilon}}^{\delta_{\epsilon}}}= \\
1+\cdots \pm \lambda^{\epsilon}(d c)^{\alpha^{\prime}+1} d
\end{gathered}
$$

Como $\alpha^{\prime} \leq 2(\epsilon-1)$, então $\alpha=\alpha^{\prime}+1 \leq 2 \epsilon$ e portanto a afirmação é verdadeira.

(4) $j_{\epsilon-1}=2, \quad j_{\epsilon}=3$

$$
\begin{gathered}
\prod_{i=1}^{\epsilon} x_{j_{\epsilon}}^{\delta_{\epsilon}}=\underbrace{\left(1+\cdots \pm \lambda^{\epsilon-1} \cdot(d c)^{\alpha^{\prime}}\right)}_{\prod_{i=1}^{\epsilon} x_{j_{i}}^{\delta_{i}}} \underbrace{\left(1+\delta_{\epsilon} \lambda(1-d) c d c(1+d)\right)}_{x_{j_{\epsilon}}^{\delta_{\epsilon}}}= \\
1+\cdots \pm \lambda^{\epsilon}(d c)^{\alpha^{\prime}+2}(1+d)
\end{gathered}
$$

Como $\alpha^{\prime} \leq 2(\epsilon-1)$, então $\alpha=\alpha^{\prime}+2 \leq 2 \epsilon$ e portanto a afirmação é verdadeira. (5) $j_{\epsilon-1}=3, \quad j_{\epsilon}=1$

$$
\begin{gathered}
\prod_{i=1}^{\epsilon} x_{j_{\epsilon}}^{\delta_{\epsilon}}=\left(1+\cdots \pm \lambda^{\epsilon-1} \cdot(d c)^{\alpha^{\prime}}(1+d)\right)\left(1+\delta_{\epsilon} \lambda d c d\right)= \\
1+\cdots \pm \lambda^{\epsilon}(d c)^{\alpha^{\prime}+1} d
\end{gathered}
$$

Como $\alpha^{\prime} \leq 2 \cdot(\epsilon-1)$, então $\alpha=\alpha^{\prime}+1 \leq 2 \cdot \epsilon$ e portanto a afirmação é verdadeira. 
(6) $j_{\epsilon-1}=3, \quad j_{\epsilon}=2$

$$
\begin{gathered}
\prod_{i=1}^{\epsilon} x_{j_{\epsilon}}^{\delta_{\epsilon}}=\left(1+\cdots \pm \lambda^{\epsilon-1} \cdot(d c)^{\alpha^{\prime}}(1+d)\right)\left(1+\delta_{\epsilon} \lambda c\right)= \\
1+\cdots \pm \lambda^{\epsilon}(d c)^{\alpha^{\prime}+1}
\end{gathered}
$$

Como $\alpha^{\prime} \leq 2(\epsilon-1)$, então $\alpha=\alpha^{\prime}+1 \leq 2 \epsilon$ e portanto a afirmação é verdadeira.

Além disso o fato de $p_{i}^{\prime}(c, d)$ não depender de $\lambda$ nos garante que $p_{i}(c, d)$ também não depende. Logo concluimos nossa demonstração.

Assim, por esta afirmação, e do fato que $\omega\left(x_{1} x_{2}, x_{1} x_{3}\right)=1$ temos:

$$
\sum_{i=1}^{\epsilon} \lambda^{i} p_{i}(c, d)=0
$$

Como o anel $R$ é infinito, se tomarmos $\epsilon+1$ elementos distintos de $R, \lambda_{1}, \ldots \lambda_{\epsilon+1}$, teremos

$$
\left(\begin{array}{llll}
1 & \lambda_{1} & \cdots & \lambda_{1}^{\epsilon} \\
1 & \lambda_{2} & \cdots & \lambda_{2}^{\epsilon} \\
\vdots & \vdots & & \vdots \\
1 & \lambda_{\epsilon+1} & \cdots & \lambda_{\epsilon+1}^{\epsilon}
\end{array}\right)\left(\begin{array}{c}
0 \\
p_{1}(c, d) \\
\vdots \\
p_{\epsilon}(c, d)
\end{array}\right)=0
$$

Como $R$ é domínio de integridade, usando o determinante de Vandermonde teremos :

$$
p_{i}(c, d)=0,
$$

para todo $i$. Em particular:

$$
p_{\epsilon}(c, d)=0
$$

Assim:

ou

$$
(d c)^{\alpha}=0
$$

e $d c$ é nilpotente de expoente $\alpha$

ou

$$
(d c)^{\alpha} d=0 \Longrightarrow(d c)^{\alpha+1}=0
$$

e $d c$ é nilpotente de expoente $\alpha+1$,

ou

$$
(d c)^{\alpha}(1+d)=0 \Longrightarrow(d c)^{\alpha}(1+d) c=0 \Longrightarrow(d c)^{\alpha+1}=0
$$


e $d c$ é nilpotente de expoente $\alpha+1$

Portanto concluimos que $d c$ é nilpotente com expoente menor ou igual a $\alpha+1$, e além do mais

$$
\alpha+1 \leq 2 \epsilon+1 \leq 4\left(\left|r_{1}\right|+\cdots+\left|r_{2 k+1}\right|\right)+1
$$

Ou seja, o grau de nilpotência não depende da escolha de $c$ e $d$. Depende somente da natureza de $\omega$.

Vamos notar por $l$ o número $\left|r_{1}\right|+\cdots+\left|r_{2 k+1}\right|$

Vamos mostrar agora que se $a^{2}=b^{2}=0$ então $b a b A$ é nil de expoente limitado

Seja $x \in A$. Fazendo $d=b x b$ e $c=a b x b a$, pelo raciocínio anterior teremos

$$
(d c)^{m}=0 \Longrightarrow(\text { bxbabxba })^{m}=0
$$

onde $m=4 l+1$.(vale notar que $c^{2}=d^{2}=0$ )

Assim $(b a b x)^{2 m+1}=b a(b x b a b x b a)^{m} b x=0$, e portanto $b a b A$ é nil de expoente limitado $8 l+3$.

Finalmente vamos mostrar que se $b c=a^{2}=0$, então $b a c A$ é nil de grau limitado.

Se $b c=0$ temos para todo $x \in A$ que $(c x b)^{2}=0$. Assim, como $a^{2}=0$, temos pelo passo anterior que cxbacxbA é nil de grau limitado $n=8 l+3$. Desse modo

$$
(b a c x)^{2 n+1}=b a(c x b a c x b a)^{n} c x=0
$$

Logo concluimos que bacA é nil de expoente limitado, sendo que tal expoente não depende de $a, b$ ou $c$, mas apenas da natureza da identidade polinomial. (é menor ou igual a $k=16 l+7$, onde $l$ é a soma dos módulos dos $r_{i}{ }^{\prime} \mathrm{s}$ )

Logo existe $k$, tal que para todo $a, b, c, \operatorname{com} b c=a^{2}=0$, o ideal a direita $b a c A$ é nil de expoente limitado menor igual a $k$.

O próximo lema nos dará condições, sobre as quais podemos garantir que todos idempotentes de uma álgebra são centrais.

Lema 2.3. Seja A uma álgebra sobre um domínio comutativo $R$. Suponha que para todo $a, b, c \in A$ tais que $a^{2}=b c=0$ tenhamos $b a c=0$. Então todo idempotente de $R^{-1} A$ é central.

\section{DEMONSTRAÇÃO:}

Seja $e$ um idempotente em $R^{-1} A$. Então para algum $r \neq 0, r \in R, f=r e \in A$

$$
(r e)^{2}=\text { rere }=r^{2} e^{2}=r^{2} e
$$


Logo

$$
f(r-f)=r e(r-r e)=r^{2} e-r^{2} e=0
$$

Se $u \in R^{-1} A$ temos:

$$
(f u(r-f))^{2}=0
$$

pois

$$
\begin{aligned}
& (\text { reu }(r-\text { re }))^{2}=(\text { reur }- \text { reure })^{2}=\left(r^{2} e u-r^{2} e u e\right)^{2}= \\
& r^{2} e u r^{2} e u-r^{2} e u r^{2} \text { eue }-r^{2} e u e r^{2} e u+r^{4} \text { eиеeue }= \\
& r^{4} \text { eиеи }-r^{4} \text { eиеие }-r^{4} \text { еиеи }+r^{4} \text { eиеие }=0
\end{aligned}
$$

Assim, por hipótese

$$
\underbrace{f}_{=b} \cdot \underbrace{(f u(r-f))}_{=a} \cdot \underbrace{(r-f)}_{=c}=0
$$

Portanto temos

$$
f(f u(r-f)) r-f(f u \underbrace{(r-f)) f}_{=f(r-f)=0}=0
$$

e consequentemente

$$
f(f u(r-f)) r=0
$$

Por outro lado

$$
\begin{gathered}
f(f u(r-f)) r=r f^{2} u(r-f)=r^{3} e^{2} u(r-f)= \\
r^{3} \text { eur }-r^{3} \text { euf }=r^{4} \text { eu }-r^{4} \text { eue. }
\end{gathered}
$$

Do fato que $r$ não é divisor de 0 vem que:

$$
e u=e u e
$$

De forma análoga concluimos:

$$
\text { eue }=u e,
$$

e portanto temos ue $=e u$ para todo idempotente de $R^{-1} A$ e para todo $u$ de $R^{-1} A$. Logo todo idempotente de $R^{-1} A$ é central.

Pelo lema acima, podemos concluir, que para que uma álgebra tenha todos os seus idempotentes centrais basta que, para todo $a, b, c \in A$, com $a^{2}=b c=0$, tenhamos $b a c=0$. Vamos mostrar com o auxílio do teorema 2.2, e do teorema de Levitski, que se a álgebra $A$ é semiprima e $U(A)$ satisfaz uma identidade de grupo, então temos esta condição satisfeita.

Antes porém vamos enunciar uma proposição que amplia o conjunto dos a's, que fazem $b a c=0$, sempre que $b c=0$. 
Proposição 2.4. Seja $R$ um anel unitário semiprimo. Suponha que para todos $b, c, x \in R$ tais que $x^{2}=b c=0$ tenhamos $b x c=0$. Então para todo elemento nilpotente $a \in R$, tal que $b c=0$, temos $b a c=0$.

\section{DEMONSTRAÇÃO:}

Vamos provar usando indução no índice de nilpotência de $a$.

Seja $a \in R$ tal que $a^{n}=0, a^{n-1} \neq 0$.

Se $x^{2}=0$, por hipótese $b x c=0$.

Hipótese de indução: Se $x \in R \operatorname{com} x^{m}=0$ e $m<n$, então $b^{\prime} x c^{\prime}=0$ sempre que $b^{\prime} c^{\prime}=0$, e $b^{\prime}$ e $c^{\prime}$ estão em $R$.

Se $a^{n}=0$ para algum $n$, então:

$$
(1-a) \cdot\left(1+a+\cdots a^{n-1}\right)=\left(1+a+\cdots a^{n-1}\right)(1-a)=1-a^{n}=1 .
$$

Logo $1-a$ é inversível.

Sejam $b, c \in R$ tal que $b c=0$ e seja $r \in R$.

Tomando $b_{1}=b(1-a)^{-1}$ e $c_{1}=(1-a) c$ temos

$$
b_{1} c_{1}=b(1-a)^{-1}(1-a) c=b c=0
$$

Assim, do fato que $(c r b)^{2}=c r b c r b=0$, teremos, por um lado:

$$
b_{1} c r b c_{1}=0 \text {. }
$$

Por outro lado, se $m \geq 2$ e $n \geq 2$ temos $m(n-1) \geq n$ e portanto:

$$
\left(a^{m}\right)^{n-1}=a^{m(n-1)}=0
$$

Assim, pela hipótese de indução $b a^{m} c=0$ para todo $m$, maior ou igual a 2 .

Donde concluimos que,

$$
b_{1} c=b\left(1+a+a^{2}+\cdots a^{n-1}\right) c=b c+b a c+\underbrace{b a^{2} c+\cdots b a^{(n-1)} c}_{=0}=b a c
$$

e

$$
b c_{1}=b(1-a) c=b c-b a c=-b a c
$$

e assim:

$$
b_{1} c r b c_{1}=(b a c r(-b a c))=-b a c r b a c .
$$


Portanto:

$$
\text { bacrbac }=0,
$$

para todo $r \in R$.

Se supormos que $b a c \neq 0$, o ideal bilateral $R b a c R=\left\{\sum r_{i} b a c s_{i} \mid r_{i}, s_{i} \in R\right\}$, será não nulo, pois bac $\in$ RbacR

E além disto será nilpotente:

$$
\begin{gathered}
\text { Sejam } x_{1}=\sum_{i=1}^{n} r_{i} \text { bacs }_{i}, \text { e } x_{2}=\sum_{j=1}^{m} t_{j} b a c w_{j}, \operatorname{com} s_{i}, r_{i}, t_{j}, w_{j} \in R . \text { Teremos: } \\
x_{1} x_{2}=\sum_{i, j} r_{i} b a c \underbrace{s_{i} t_{j}}_{=l_{i, j} \in R} b a c w_{j}= \\
\sum_{i, j} r_{i} \underbrace{b a c l_{i, j} b a c}_{=0} w_{j}=0,
\end{gathered}
$$

Logo $R b a c R$ é não nulo, e nilpotente de expoente 2 , o que contraria o fato de $R$ ser semiprimo.

Portanto $b a c=0$, para todo elemento nilpotente $a$ pertencente a $R$, e todo $b$, e $c$ tal que $b c=0$.

Teorema 2.5 (Levitzky). Seja $R$ um anel. E seja $\mathcal{H}(R)$, o conjunto:

$$
\mathcal{H}(R)=\{a \in R \mid a R \text { é ideal nil de exponte limitado }\}
$$

Se $R$ é um anel semiprimo então $\mathcal{H}(R)=0$.

DEMONSTRAÇÃO: ver pag 46, corolário 1.6.26 de [Row80].

Enfim enunciaremos, e demonstraremos, o teorema principal do capítulo.

Teorema 2.6. Seja A uma álgebra semiprima unitária sobre um dominio comutativo infinito $R$. Se $U(A)$ satisfaz uma identidade de grupo, então para todos $b, c \in A$ tais que $b c=0$ e todo elemento nilpotente $a \in A$ tem-se bac $=0$. Além disso todo idempotente de $R^{-1} A$ é central.

\section{DEMONSTRAÇÃO:}

Sejam $a, b, c \in A$, tal que $a^{n}=b c=0$, para algum $n \in \mathbb{N}$. Vamos primeiramente mostrar que: 


$$
a^{2}=b c=0 \Longrightarrow b a c=0:
$$

Pelo teorema 2.2 temos que bac $A$ é um ideal nil a direita de expoente limitado.

Se $b a c \neq 0$, temos $\mathcal{H}(A) \neq(0)$. Pelo teorema de Levitzki, $A$ não seria semiprimo. Assim temos $b a c=0$.

Pela proposição 2.4 temos que para todo elemento nilpotente $a \in A$ bac $=0$.

Além disso pelo lema 2.3 temos que todos idempotentes de $R^{-1} A$ são centrais, concluindo nossa demonstração.

Este resultado será fundamental na demonstração do principal teorema do próximo capítulo, no caso em que $F[G]$ for um anel semiprimo. Indiretamente também terá importância na demonstração do caso em que $N(F[G]$ ), (que será definido posteriormente), é nilpotente. 


\section{Capítulo 3}

\section{Caso Geral.}

\subsection{Introdução}

Nosso objetivo neste capítulo será demonstrar o seguinte teorema:

Teorema 3.1 (Giambruno, Sehgal, Valenti). Sejam $F$ um corpo infinito e $G$ um grupo de torção. Se $U(F[G])$ satisfaz uma identidade de grupo então $F[G]$ satisfaz uma identidade polinomial.

Vamos antes fixar algumas notações.

Usaremos a letra $p$ para designar a característica do corpo $F$. Usaremos $(u, v)$ para denotar o comutador multiplicativo $u^{-1} v^{-1} u v$, e $[x, y]$ para denotar o comutador aditivo $x y-y x$.

Como no capítulo 1 , se $N$ é um subgrupo normal de $G$, denotaremos por $\Delta(G, N)$, o kernel da projeção canônica $\pi$,

$$
\pi: F[G] \longrightarrow F\left[\frac{G}{N}\right]
$$

e por $\Delta(G, G)$, o ideal de aumento que corresponde ao kernel da projeção canônica $\pi^{\prime}$

$$
\pi^{\prime}: F[G] \longrightarrow F
$$

Vamos definir também

$$
P=\left\{g \in G \mid o(g)=p^{k} \text { para algum } k \in \mathbb{N}\right\}
$$




$$
Q=\{g \in G \mid p \nmid o(g)\} .
$$

Os elementos de $P$ e $Q$ serão chamados de $p$-elementos, e $p^{\prime}$-elementos respectivamente.

Proposição 3.2. Seja $p$ um inteiro primo, $G$ um grupo de torção e $P$ e $Q$ como acima. Então $G=P Q$

\section{DEMONSTRAÇÃO:}

Seja $g \in G$, e seja $m$ a ordem de $g$. Claramente $m$ pode ser escrito na forma $m=p^{k} a \operatorname{com}(a, p)=1$.

Usando o teorema de Bezout temos que existem $r$ e $s$ inteiros tais que

$$
1=r p^{k}+s a
$$

Assim

$$
g=g^{r p^{k}+s a}=g^{s a} g^{r p^{k}}
$$

Se observarmos que

$$
\left(g^{s a}\right)^{p^{k}}=g^{s \cdot a p^{k}}=g^{s m}=1
$$

e que

$$
\left(g^{r p^{k}}\right)^{a}=g^{r \cdot a p^{k}}=g^{r m}=1
$$

Temos que $o\left(g^{s a}\right)$ divide $p^{k}$, e portanto $g^{s a} \in P$. Também concluimos que $p$ não divide a ordem de $g^{r p^{k}}$ pois senão dividiria $o(a)$. Logo $g^{r p^{k}} \in Q$.

Portanto concluimos que $G=P Q$

Vamos introduzir a noção de grupo $p$-abeliano.

Definição 3.3. Seja $G$ um grupo. Dizemos $G$ é p-abeliano se o seu comutador é um p-grupo finito.

Proposição 3.4. Sejam $G$ e $P$ como no teorema anterior. Se $G^{\prime}$ é um p-grupo, então $P$ é subgrupo de $G$.

\section{DEmonstraÇÃo:}

Claramente $1 \in P$, (basta tomar $k=0$ ), e se $o(g)=p^{k}$, temos $o\left(g^{-1}\right)=p^{k}$. Logo basta mostrar que se $o\left(g_{1}\right)=p^{k_{1}}$, e $o\left(g_{2}\right)=p^{k_{2}}$ então existe $k_{3}$ tal que $o\left(g_{1} g_{2}\right)=p^{k_{3}}$.

Vamos notar por $\bar{g}$, o elemento $g \cdot G^{\prime} \in G / G^{\prime}$, e seja $k=k_{1}+k_{2}$.

Como $G / G^{\prime}$ é abeliano teremos:

$$
\overline{\left(g_{1} g_{2}\right)^{p^{k}}}=\left(\overline{g_{1} g_{2}}\right)^{p^{k}}=\left(\overline{g_{1}} \overline{g_{2}}\right)^{p^{k_{1}+k_{2}}}=\overline{g_{1}} p^{k_{1}+k_{2}}{\overline{g_{2}}}^{p^{k_{1}+k_{2}}}=\overline{1}
$$


$\operatorname{Logo}\left(g_{1} g_{2}\right)^{p^{k_{1}+k_{2}}} \in G^{\prime}$.

Como $G^{\prime}$ é p-grupo, para todo $a \in G^{\prime}$, existe $k_{4}$ tal que $a^{p^{k_{4}}}=1$.

Logo se tomarmos $k_{3}=k_{1}+k_{2}+k_{4}$, teremos $\left(g_{1} g_{2}\right)^{p^{k_{3}}}=1$.

Vamos agora introduzir alguns conceitos referentes a álgebras livres.

Seja $F$ um corpo de característica $p$. Notaremos por $F\left\langle x_{1}, x_{2}, \ldots, x_{n}\right\rangle$ a $F$-álgebra livre em $n$ variáveis, por $\mathcal{A}=F\left\langle x_{1}, x_{2}, \ldots, x_{n}\right\rangle[[t]]$, o anel das séries de potências na variável comutativa $t$ sobre o álgebra livre $F\left\langle x_{1}, x_{2}, \ldots, x_{n}\right\rangle$.

Proposição 3.5. Sejam $F$ um corpo de característica $p>0, e \mathcal{A}=F\left\langle x_{1}, x_{2} \ldots x_{n}\right\rangle[[t]]$. Então:

1. $\left(1+x_{i} t\right)^{p^{\alpha_{i}}}=1+\left(x_{i} t\right)^{p^{\alpha_{i}}}$;

2. $\left(1+\left(x_{i} t\right)^{\beta}\right)$ é inversivel para todo $\beta$ em $\mathbb{N}$, com inverso,

$$
1+\sum_{k=1}^{\infty}(-1)^{k} x_{i}{ }^{\beta k} t^{\beta k}
$$

3. $\left(1+\left(x_{i} t\right)^{\beta}\right)^{n}=1+n\left(x_{i} t\right)^{\beta}+\left(x_{i} t\right)^{2 \beta} q_{n}\left(\left(x_{i} t\right)^{\beta}\right)$, onde $q_{n}$ é um polinômio (se $n \geq 0)$, ou uma série de potências (se $n<0)$ com coeficientes em $F$.

Demonstração:

(1)-Usando o teorema do binômio, mais o fato que se $p$ é primo $p$ divide $\left(\begin{array}{c}p^{\alpha_{i}} \\ k\end{array}\right)$, para $1 \leq k \leq p^{\alpha_{i}}-1$, concluimos que:

$$
\left(1+x_{i} t\right)^{p^{\alpha_{i}}}=1+\left(x_{i} t\right)^{p^{\alpha_{i}}} .
$$

$$
\begin{gathered}
\left(1+\left(x_{i} t\right)^{\beta}\right)\left(1+\sum_{k=1}^{\infty}(-1)^{k} x_{i}{ }^{\beta k} t^{\beta k}\right)= \\
1+\sum_{k=1}^{\infty}\left(x_{i}^{\beta k}-x_{i}{ }^{\beta k}\right) t^{\beta k}=1 .
\end{gathered}
$$

Analogamente:

$$
\left(1+\sum_{k=1}^{\infty}(-1)^{k} x_{i}^{\beta k} t^{\beta k}\right)\left(1+\left(x_{i} t\right)^{\beta}\right)=1
$$


Se $n \geq 0$, a conclusão segue pelo teorema do binômio.

Vamos ao caso $n<0$ :

Seja $m=-n$.

Provaremos por indução em $m$.

Se $m=1$ a afirmação segue do item 2 isto é

$$
q_{-1}\left(\left(x_{i} t\right)^{\beta}\right)=\sum_{k=0}^{\infty}(-1)^{k} \cdot\left(x_{i} t\right)^{\beta k}
$$

Pela hipótese de indução temos

$$
\left(1+\left(x_{i} t\right)^{\beta}\right)^{-(m-1)}=\left(1-(m-1)\left(x_{i} t\right)^{\beta}+\left(x_{i} t\right)^{2 \beta} q_{n+1}\left(\left(x_{i} t\right)^{\beta}\right),\right.
$$

onde $q_{n+1}\left(\left(x_{i} t\right)^{\beta}\right)$ é uma série de potências. Assim temos:

$$
\begin{gathered}
\left(1+\left(x_{i} t\right)^{\beta}\right)^{-m}=\left[1-\left(x_{i} t\right)^{\beta}+\left(x_{i} t\right)^{2 \beta} q_{-1}\left(\left(x_{i} t\right)^{\beta}\right)\right]\left[1-(m-1)\left(x_{i} t\right)^{\beta}+\left(x_{i} t\right)^{2 \beta} q_{n+1}\left(\left(x_{i} t\right)^{\beta}\right)\right]= \\
1-\left(x_{i} t\right)^{\beta}-(m-1)\left(x_{i} t\right)^{\beta}+\left(x_{i} t\right)^{2 \beta} q_{n}\left(\left(x_{i} t\right)^{\beta}\right)= \\
1-m\left(x_{i} t\right)^{\beta}+\left(x_{i} t\right)^{2 \beta} q_{n}\left(\left(x_{i} t\right)^{\beta}\right)
\end{gathered}
$$

onde

$$
q_{n}\left(\left(x_{i} t\right)^{\beta}\right)=m-1+\left[1-\left(x_{i} t\right)^{\beta}\right] q_{n+1}\left(\left(x_{i} t\right)^{\beta}\right)+q_{-1}\left(\left(x_{i} t\right)^{\beta}\right) \cdot\left(1+\left(x_{i} t\right)\right)^{n+1}
$$

E assim concluimos a demonstração.

Definição 3.6. Seja $h\left(x_{1}, \ldots, x_{n}, t\right)=\alpha x_{i_{1}}{ }^{{ }} \cdots x_{i_{k}}{ }^{{ }}{ }^{\beta} t^{\beta}$ um monômio não constante do anel das séries de potências de $t$ sobre a álgebra livre, $F\left\langle x_{1}, \ldots x_{n}\right\rangle[[t]],(\alpha \in F)$, tal que $i_{j} \neq i_{j+1}$, para todo $j \leq k-1$. Nesta situação diremos que $k$ é o comprimento silábico de $h$. (Se $h$ for do tipo $\alpha t^{r}$, diremos que $h$ tem comprimento silábico igual a 0$)$

Vale notar que sempre podemos escrever um monômio $h$ não constante de $F\left\langle x_{1}, \ldots x_{n}\right\rangle$ na forma acima de maneira única, garantindo assim que comprimento silábico está bem definido.

Vamos demonstrar agora uma proposição, devida a Wilhelm Magnus. (ver [MKS76].)

Proposição 3.7 (Magnus). Seja $F$ um corpo de característica $p>0, e \mathcal{A}=$ $F\left\langle x_{1}, \ldots x_{n}\right\rangle[[t]]$. Os elementos $1+x_{1} t, \ldots, 1+x_{n} t$, geram um grupo livre no grupo das unidades de $\mathcal{A}$. 


\section{DemonstraçÃo:}

Vimos no item 2 da proposição 3.5 , que $1+x_{1} t, \ldots, 1+x_{n} t$ são elementos inversíveis de $\mathcal{A}$. Basta mostrar que qualquer produto $\omega$ da forma,

$$
\omega=\left(1+x_{i_{1}} t\right)^{r_{1}} \cdots\left(1+x_{i_{k}} t\right)^{r_{k}},
$$

com $i_{j} \neq i_{j+1}$, para $j \leq k-1$, e $r_{j} \neq 0$, para todo $j$, é diferente de 1 .

Vamos escrever $r_{j}=\alpha_{j} \cdot \beta_{j}$, onde $\alpha_{j}$ é uma potência de $p$, e $\left(p, \beta_{j}\right)=1$.

Pelo item 1 da proposição 3.5 , temos:

$$
\omega=\left(1+\left(x_{i_{1}} t\right)^{\alpha_{1}}\right)^{\beta_{1}} \cdots\left(1+\left(x_{i_{1}} t\right)^{\alpha_{k}}\right)^{\beta_{k}} .
$$

E pela parte 3 da mesma proposição temos,

$$
\omega=\left(1+\beta_{1}\left(x_{i_{1}} t\right)^{\alpha_{1}}+Q_{1}\right) \cdots\left(1+\beta_{k}\left(x_{i_{1}} t\right)^{\alpha_{k}}+Q_{k}\right)
$$

onde os $Q_{j}$ 's são somas de monômios onde $t$ tem grau maior ou igual a $2 \alpha_{j}$.

Segue daí que o único monômio resultante do produto que tem comprimento silábico $k$, e cujo grau de $t$ é igual a $s=\sum_{i=1}^{k} \alpha_{k}$ será

$$
\beta_{1} \cdots \beta_{k} x_{i_{1}}{ }^{\alpha_{1}} \cdots x_{i_{k}}{ }^{\alpha_{k}} t^{s}
$$

Como $\left(\beta_{j}, p\right)=1$, para todo $j$, temos,

$$
\omega=1+\cdots+\beta_{1} \cdots \beta_{k} x_{i_{1}}{ }^{{ }_{1}} \cdots x_{i_{k}}{ }^{\alpha_{k}} t^{s}+\cdots \neq 1 .
$$

E portanto concluimos, que $1+x_{1} t, \ldots, 1+x_{n} t$ geram um grupo livre de posto $\mathrm{n}$ no grupo de unidades de $\mathcal{A}$.

Lema 3.8 (Giambruno,Sehgal,Valenti). Seja $G$ um grupo finito e $F$ um corpo infinito tal que $U(F[G])$ satisfaz uma identidade de grupo. Se a característica de $F$ é $p>0$ então $G$ é p-abeliano. Se $F$ é um corpo de característica $0 G$ será abeliano.

\section{DEMONSTRAÇÃO:}

O fato de $G$ ser finito implica que $F[G]$ é artiniano a esquerda, pois todo ideal a esquerda de $F[G]$, pode ser visto como um espaço vetorial de dimensão finita sobre $F$. Portanto, se um ideal está contido propriamente em outro, então sua dimensão 
será menor que a daquele que o contém. Como $F[G]$ tem dimensão finita sobre $F$, em algum momento a cadeia para de decrescer.

Seja $\mathcal{J}$ o radical de Jacobson de $F[G]$. Então, $\mathcal{J}$ é nilpotente e temos o epimorfismo canônico:

$$
\pi: F[G] \longrightarrow \frac{F[G]}{\mathcal{J}}
$$

Como o radical de Jacobson é nilpotente, segue pela proposição 1.26 que $U\left(\frac{F[G]}{\mathcal{J}}\right)$ satisfaz a uma identidade de grupo.

Do fato de $F[G]$ ser artiniano a esquerda temos que $\frac{F[G]}{\mathcal{J}}$ também será.

Como $\frac{F[G]}{\mathcal{J}}$ é semiprimitivo e artiniano a esquerda pelo teorema 1.13 , será semisimples.

Usando o teorema de Artin-Wedderburn (ver teorema 1.14):

$$
\frac{F[G]}{\mathcal{J}}=\bigoplus_{i \in I} M_{n_{i}}\left(D_{i}\right)
$$

Como $\frac{F[G]}{\mathcal{J}}$ é semiprimitivo, pela proposição 1.13, será semiprimo e usando o teorema 2.6 temos que todo idempotente de $\frac{F[G]}{\mathcal{J}}$ é central. E portanto concluimos que $n_{i}=1$ para todo $i$. Assim,

$$
\frac{F[G]}{\mathcal{J}}=\bigoplus_{i \in I} D_{i} .
$$

onde cada $D_{i}$ é um anel com divisão, com $F$ contido em seu centro.

Além disso, do fato de $G$ ser finito temos que $\left[D_{i}: F\right]<\infty$.

Pelo lema 1.16 cada $D_{i}$ deve ser comutativo. Caso contrário irá conter um grupo livre de posto 2 , e portanto seu grupo de unidades não irá satisfazer nenhuma identidade de grupo.

Assim $\frac{F[G]}{\mathcal{J}}$ é soma direta de corpos, logo é comutativo. Logo, para todo $x, y$ pertencentes a $U(F[G])$

$$
\pi(x, y)=(\pi(x), \pi(y))=1=\pi(1)
$$

e portanto,

$$
(x, y)=1(\bmod \mathcal{J}),
$$

Assim concluimos que $G^{\prime} \subseteq 1+\mathcal{J}$, logo 


$$
\Delta\left(G^{\prime}\right) \subseteq \mathcal{J}
$$

Assim, $\Delta\left(G^{\prime}\right)$ será nilpotente.

Se $p>0$, pela proposição 1.23 temos que $G^{\prime}$ é um $p$-grupo finito. Se $p=0$, temos pela mesma proposição que $G^{\prime}=(1)$, e portanto $G$ é abeliano.

Podemos remover a hipótese de $G$ ser finito, e enunciar uma espécie de recíproca:

Lema 3.9 (Giambruno, Sehgal, Valenti). Seja $G$ um grupo, e seja $F$ um corpo de característica $p>0$. Se $G$ é p-abeliano então $U$ satisfaz uma identidade de grupo, e $F[G]$ satisfaz a identidade polinomial $[x, y]^{p^{m}}=0$ para algum $m \in \mathbb{N}$.

DEMONSTRAÇÃO:

Seja $\pi$,

$$
\pi: F[G] \longrightarrow F\left[\frac{G}{G^{\prime}}\right]
$$

o epimorfismo canônico. Sejam $u$ e $v$ elementos de $U(F[G])$.

Como $\frac{G}{G^{\prime}}$ é abeliano, temos:

$$
\pi\left(u v u^{-1} v^{-1}\right)=(\pi(u), \pi(v))=1
$$

e portanto concluimos que $1-(u, v)$ pertence ao kernel de $\pi$.

Pelo corolário 1.24 temos que o kernel de $\pi$ é nilpotente. Assim existe $k$, tal que

$$
(1-(u, v))^{p^{k}}=0 .
$$

Por outro lado,

$$
(1-(u, v))^{p^{k}}=1-(u, v)^{p^{k}}
$$

Donde concluimos que $(u, v)^{p^{k}}=1$, e portanto $U(F[G])$ satisfaz a identidade de grupo

$$
\omega(x, y)=(x, y)^{p^{k}}=1
$$

Além disso se $x, y \in F[G]$ :

$$
[x, y]=\sum c[g, h]
$$

onde $c \in F$ e $g, h \in G$

$$
=\sum c g h-c h g=\sum \operatorname{cgh}\left(1-h^{-1} g^{-1} h g\right)=\sum \underbrace{c g h}_{\in F[G]} \underbrace{(1-(g, h))}_{\in \Delta\left(G^{\prime}\right)}
$$


Logo $[x, y] \in F[G] \cdot\left(\Delta\left(G^{\prime}\right)\right)$. Da proposição 1.23 , e do fato que $G^{\prime}$ é $p$ grupo segue que existe $k$, tal que $[x, y]^{p^{k}}=0$

Assim demonstramos que $F[G]$ satisfaz uma identidade polinomial.

\subsection{O grupo $\Phi(G)$}

Nesta seção estudaremos o subgrupo de conjugação finita de $G$, que iremos notar o grupo $\Phi(G)$. Ele irá ocupar um lugar importante na demonstração do principal teorema deste capítulo.

Definição 3.10. Seja $G$ um grupo e $g$, um elemento de $G$. A classe de conjugação de $g$ em $G$, que notamos por $\operatorname{cl}_{G}(g)$ é o conjunto

$$
\operatorname{cl}_{G}(g)=\left\{h^{-1} g h \mid h \in G\right\} .
$$

Um elemento $g$ de $G$ é dito de conjugação finita em $G$ se $\left|\operatorname{cl}_{G}(g)\right|<\infty$. Representaremos por $\Phi(G)$ o conjunto dos elementos de conjugação finita em $G$.

Teorema 3.11. $\Phi(G)$ é subgrupo normal de $G$

DEMONSTRAÇÃO:

Inicialmente vamos demonstrar que $\Phi(G)$ é subgrupo de $G$ :

Certamente $1 \in \Phi(G)$ e $|\operatorname{cl}(g)|=\left|\operatorname{cl}\left(g^{-1}\right)\right|$. Vamos mostrar que se $g, h \in \Phi(G)$ então $g h \in \Phi(G)$. De fato

$$
\operatorname{cl}(g h)=\left\{k^{-1} g h k \mid k \in G\right\}=\left\{k^{-1} g k k^{-1} h k \mid k \in G\right\},
$$

e

$$
|\operatorname{cl}(g h)| \leq|\operatorname{cl}(g)| \cdot|\operatorname{cl}(h)|<\infty .
$$

Portanto $g h \in \Phi(G)$, e $\Phi(G)$ é subgrupo de $G$.

A normalidade de $\Phi(G)$, segue do fato que $\operatorname{cl}\left(h^{-1} g h\right)=\operatorname{cl}(g)$, para todo $g \in \Phi(G)$, e todo $h \in G$.

Se $G=\Phi(G)$, ou equivalentemente, todo elemento de $G$ tem conjugação finita, dizemos que $G$ é um grupo de conjugação finita.

Seja $G$ um grupo, $g$ um elemento de $G$ e seja $C_{G}(g)=\{h \in G \mid h g=g h\}$, o centralizador de $g$ em $G$. Então:

$$
\left|\mathrm{cl}_{G}(g)\right|=\left[G: C_{G}(g)\right]
$$


Este resultado nos diz que $g \in \Phi(G)$ se e somente se $\left[G: C_{G}(g)\right]<\infty$. Usaremos esta equivalência para demonstrar o seguinte resultado:

Proposição 3.12. Seja $G$ um grupo de torção. Então $\Phi(G)$ é localmente finito.

\section{DEMONSTRAÇÃO:}

Seja $G_{0}$ um subgrupo de $\Phi$ com um número finito de geradores, $G_{0}=\left\langle h_{1}, \ldots h_{n}\right\rangle$. Comỏ $G_{0}$ é um grupo de conjugação finita, temos,

$$
\left[G_{0}: C_{G_{0}}\left(h_{i}\right)\right]<\infty
$$

para todo $h_{i}$ e portanto,

$$
\left[G_{0}: \bigcap C_{G_{0}}\left(h_{i}\right)\right]<\infty
$$

Mas $\cap C_{G_{0}}\left(h_{i}\right)=Z\left(G_{0}\right), \operatorname{logo}$

$$
\left[G_{0}: Z\left(G_{0}\right)\right]<\infty
$$

Da desigualdade acima, e da proposição 1.6, podemos concluir que $Z\left(G_{0}\right)$ é finitamente gerado.

Ora, $Z\left(G_{0}\right)$ é finitamente gerado, abeliano e de torção, logo $Z\left(G_{0}\right)$ é finito. Portanto $G_{0}$ é finito. Logo $\Phi(G)$ será localmente finito. $\Phi(G)$.

Quando não houver o risco de interpretações dúbias, notaremos por $\Phi$ o conjunto

Notaremos por $\Phi_{p}(G)$, o subgrupo de $G$, gerado pela intersecção dos conjuntos $\Phi(G)$, e $P=\left\{g \in G \mid o(g)=p^{k}\right.$ para algum $\left.k \in \mathbb{N}\right\}$, isto é,

$$
\Phi_{p}=\langle P \cap \Phi\rangle \text {. }
$$

Proposição 3.13. Seja $G$ um grupo de torção. Se $\Phi_{p}(G)$ é finito então $\Phi\left(\frac{G}{\Phi_{p}(G)}\right)$, não contém p-elementos.

\section{DEMONSTRAÇÃO:}

Vamos notar por $N$ o grupo $\Phi_{p}(G)$.

Seja $\bar{h}=h N \in \Phi\left(\frac{G}{\Phi_{p}(G)}\right)$, vamos mostrar que $p$ não divide $o(h N)$. 
Vamos primeiramente mostrar que $h \in \Phi(G)$ sempre que $h N \in \Phi\left(\frac{G}{\Phi_{p}(G)}\right)$ : De fato, se $h N \in \Phi\left(\frac{G}{\Phi_{p}(G)}\right)$ então o conjunto

$$
\operatorname{cl}(h N)=\left\{g^{-1} h g N \mid g N \in \frac{G}{N}\right\}
$$

é finito. Como $N$ é um conjunto finito, o conjunto união de $\operatorname{cl}(h N)$, que notaremos por $S$

$$
S=\left\{x \mid x \in g^{-1} h g N, g \in G\right\},
$$

também será finito. Logo se $q \in G$ é tal que existe $g$ com $q=g^{-1} h g$, claramente $q \in S$, e assim

$$
c l(h)=\left\{g^{-1} h g \mid g \in G\right\}
$$

é finito. Assim temos que $h \in \Phi(G)$.

Vamos mostrar agora que $p$ não divide $o(h N)$ :

Seja $o(h)=p^{k} m$, onde $k$ é um número natural e $(m, p)=1$.

Pela proposição 3.2 existem $h_{1}$ e $h_{2}$, tais que

$$
h=h_{1} h_{2}=h_{2} h_{1}
$$

$\mathrm{e}$

$$
h_{1}{ }^{m}=h_{2}^{p^{k}}=1
$$

em particular temos que $h_{2} \in N$,e assim:

$$
(h N)^{m}=\left(h_{1} N h_{2} N\right)^{m}=\left(h_{1} N\right)^{m} \underbrace{\left(h_{2} N\right)^{m}}_{h_{2} \in N}=h_{1}{ }^{m} N=N
$$

Logo $o(h N)$ divide $m$, e portanto $p$ não irá dividir a ordem de $h N$.

Logo $\Phi\left(\frac{G}{\Phi_{p}(G)}\right)$ não tem $p$-elementos.

Lema 3.14 (Passman). Suponha que $F$ é um corpo de característica $p>0$. Então $F[G]$ é semiprimo se e somente se $\Phi(G)$ é um $p^{\prime}$-grupo.

DemonstraÇÃo: ver pag 131, teorema 2.13 do capítulo 4 de [Pas77].

Vamos notar por $N=N(F[G])$ a soma de todos ideais nilpotentes de $F[G]$. Claramente $N$ será um ideal nil de $F[G]$.

Lema 3.15 (Passman). Seja $F$ um corpo de característica $p>0$. Então $N(F[G])$ é nilpotente se e somente se $\Phi_{p}$ é finito.

DemonstraÇÃo: ver pag 311, teorema 1.12 do capítulo 8 de [Pas77]. 


\subsection{Relações entre o grupo $G$ e as identidades po- linomiais satisfeitas por $F[G]$}

Nesta seção analisaremos algumas relações entre o grupo $G$ e o fato de $F[G]$ satisfazer uma identidade polinomial. (Ou uma identidade polinomial generalizada.). Enunciaremos alguns teoremas, que por terem demonstrações trabalhosas não serão demonstrados. Indicaremos ao leitor, porém, onde encontrar tais demonstrações.

Lema 3.16 (Isaacs-Passman). 1. Sejam $G$ um grupo, e F um corpo de característica 0 . São equivalentes:

(i) $F[G]$ satisfaz uma identidade polinomial;

(ii) $G$ contém um subgrupo $A$ abeliano de indice finito;

(iii) $G$ contém um subgrupo normal $N$, abeliano de índice finito.

2. Sejam G um grupo e F um corpo de característica $p>0$. São equivalentes:

(i) $F[G]$ satisfaz uma identidade polinomial;

(ii) $G$ contém um subgrupo $A$ p-abeliano de indice finito;

(iii) $G$ contém um subgrupo normal $N, p$ - abeliano de indice finito.

DEMONSTRAÇÃo: ver pp 196 e 197, corolários 3.8 e 3.9 do capítulo 5 de [Pas77].

Teorema 3.17 (Passman). Seja $G$ um grupo,e $F$ um corpo de característica 0. Então $F[G]$ é semiprimo.

DemonstraÇÃo: ver teorema 2.12 do capítulo 4 de [Pas77].

Vamos enunciar dois resultados que relacionam mais estreitamente identidades polinomiais e o grupo $\Phi(G)$.

Proposição 3.18 (Passman). Se $F[G]$ satisfaz uma identidade polinomial de grau $n$, então $[G: \Phi(G)] \leq n / 2$, e $\left|(\Phi(G))^{\prime}\right|<\infty$.

DemonstraÇÃo: ver pag 189, do teorema 2.14, do capítulo 5 de [Pas77]

Teorema 3.19. Seja $F[G]$ um anel de grupo. São equivalentes:

1. $[G: \Phi(G)]<\infty$ e $\left|(\Phi(G))^{\prime}\right|<\infty$; 
2. $F[G]$ possui um idempotente e diferente de 0 , tal que eF $[G]$ e satisfaz uma identidade polinomial;

3. $F[G]$ satisfaz uma identidade polinomial generalizada não degenerada.

DemonstraÇÃo: ver [Pas71].

\subsection{Demonstração do Teorema Principal}

Dedicaremos esta seção a demonstração do teorema enunciado no começo do capítulo:

Teorema (Giambruno, Sehgal, Valenti). Sejam $F$ um corpo infinito e $G$ um grupo de torção. Se $U(F[G])$ satisfaz uma identidade de grupo então $F[G]$ satisfaz uma identidade polinomial.

DEMONSTRAÇÃo: Dividiremos a demonstração em 3 casos:

- 1- $F[G]$ é semiprimo:(isto é, $N=0$ )

Seja $p=0$

Seja $y \in G$, com $o(y)=m$. Tomando $e=\frac{\hat{y}}{m}=\frac{1+y+\cdots+y^{m-1}}{m}$, e será idempotente. Pelo lema 2.3, e é central.Logo

$$
g \hat{y}=\hat{y} g, \quad \forall g \in G
$$

e portanto

$$
\begin{gathered}
g \hat{y} g^{-1}=\hat{y} \Longrightarrow \\
1+g y g^{-1}+\cdots+g y^{m-1} g^{-1}=1+y+\cdots+y^{m-1}
\end{gathered}
$$

Daqui concluimos que existe um $j$ tal que $g y g^{-1}=y^{j}$ e portanto $\langle y\rangle \triangleleft G$ para todo $y \in G$.

Como todo subgrupo cíclico de $G$ é normal podemos concluir pelo teorema de Dedekind-Baer que ou $G$ é abeliano ou é hamiltoniano. Se $G$ for Hamiltoniano $Q_{8} \leq$ $G$, e portanto $U\left(F\left[Q_{8}\right]\right)$ satisfará uma identidade de grupo. Pelo lema $3.8 Q_{8}$ será abeliano, absurdo.

Logo concluimos que $G$ é abeliano e portanto $F[G]$ satisfaz a identidade polinomial

$$
g(x, y)=x y-y x=0
$$


Seja $p>0$ :

Sejam $P$ e $Q$ os conjuntos:

$$
Q=\{g \in G \mid p \nmid \not o(g)\}
$$

e

$$
P=\left\{g \in G \mid o(g)=p^{k} \text { para algum } k\right\} .
$$

Usando raciocínio análogo ao caso anterior concluimos que para todo $y$ pertencente a $Q,\langle y\rangle \triangleleft G$. Vamos mostrar agora que $P=(1)$, e portanto $G=Q$.

Vamos inicialmente demonstrar que para todo $h \in P,\langle h\rangle \triangleleft P$.

Seja $g \in P$, com $o(g)=p^{k}$. Temos $(1-g)^{p^{k}}=1-g^{p^{k}}=0$. Além disso

$$
\hat{h}(1-h)=0
$$

Usando a proposição 2.4 temos:

$$
\begin{gathered}
\hat{h}(1-g)(1-h)=0 \\
(\hat{h}-\hat{h} g)(1-h)=\underbrace{\hat{h}(1-h)}_{=0}-\hat{h} g+\hat{h} g h=0
\end{gathered}
$$

Donde concluimos

$$
g+h g+\cdots+h^{n-1} g=g h+\cdots+h^{n-1} g h,
$$

e portanto existirá um $k$ tal que,

$$
h^{k}=g h g^{-1} .
$$

Afirmamos que para todo $q$ em $Q$, e todo $h$ em $P$, temos $q h q^{-1}=h$.

Como $\langle q\rangle \triangleleft G$ o subgrupo $H=\langle q, h\rangle$ é finito pois $\langle q\rangle$ e $\langle h\rangle$ são finitos, e para todo $r$ existe $s$ tal que $h q^{r}=q^{s} h$ e Portanto $|\langle q, h\rangle| \leq o(q) \cdot o(h)$.

Do fato de $U(F[G])$ satisfazer uma identidade de grupo, concluimos que $U(F[H])$ também satisfaz, Pelo lema 3.8, $H$ é $p$-abeliano.

Assim

$$
(q, h)=q^{-1} \underbrace{h^{-1} q h}_{\in\langle q\rangle}=q^{-1} q^{r}
$$

para algum $r$ e daqui segue que $(q, h) \in\langle q\rangle$. 
Desse modo conseguimos um elemento em $\langle q\rangle$ de ordem $p^{s}$, para algum $s$. Logo este elemento é a identidade do grupo.

Portanto $(q, h)=1$ e $q h q^{-1}=h$, para todo $h \in P$, e para todo $q \in Q$.

Da proposição 3.2 temos que $G=P Q$, e portanto temos $\langle h\rangle \triangleleft G$.

Desse modo podemos concluir que $|c l(h)|<o(h)<\infty$. Logo $h \in \Phi(G)$ e como $F[G]$ é semiprimo, por 3.14, $\Phi$ é um $p^{\prime}$-grupo. Logo $P=(1)$, e portanto $G=Q$.

Pelo teorema de Dedekind-Baer, $G=Q$ será abeliano ou hamiltoniano.

Se $p=2 Q$ não pode ter elementos de ordem 2. Logo $Q \neq Q_{8} \times A \times E\left(Q_{8}\right.$ é 2-grupo). Assim $Q$ é abeliano.

Como $U(F[G])$ satisfaz uma identidade de grupo, $U\left(F\left[Q_{8}\right]\right)$ também a satisfaz.

Por outro lado, se $p \neq 2$, pelo lema $3.8 Q_{8}$ deverá ser um grupo $p$-abeliano, ou seja seu derivado deve ser $p$-grupo. Mas $Q_{8}^{\prime}=\{1,-1\}$ é um 2-grupo. Logo $Q_{8} \not \leq Q$, donde concluimos que $Q$ é abeliano.

Como $G$ é abeliano $F[G]$ satisfaz a identidade polinomial

$$
g(x, y)=x y-y x
$$

E assim concluimos o caso semi-primo.

Pelo teorema 3.17 , podemos supor $p>0$, daqui em diante.

- $2-N$ é nilpotente e diferente de 0 :

Afirmamos que $\Phi_{p}(G)=\langle P \cap \Phi\rangle$ é normal em $G$.

De fato, se $h \in \Phi_{p}, h=h_{1} \cdot h_{2} \cdots h_{k}$, com $h_{1}, \ldots, h_{k} \in P \cap \Phi$.

Portanto

$$
g^{-1} h g=g^{-1} h_{1} g \cdot g^{-1} h_{2} g \cdots g^{-1} h_{k} g
$$

Mas $o\left(g^{-1} h_{i} g\right)=o\left(h_{i}\right)$ e $c l\left(g^{-1} h_{i} g\right)=c l\left(h_{i}\right)$. Assim, para cada $i$, temos $g^{-1} h_{i} g \in$ $P \cap \Phi$, e portanto $g^{-1} h g \in \Phi_{p}(G)$, o que prova a afirmação.

Agora, como $N$ é nilpotente, pelo lema $3.15 \Phi_{p}(G)$ é grupo finito. Além disso, segue do lema 3.8, que $\left(\Phi_{p}(G)\right)^{\prime}$, é p-grupo e portanto da proposição $3.4, \Phi_{p}(G)$ é p-grupo. Pela proposição 1.27 , temos que $U\left(F\left[\frac{G}{\Phi_{p}(G)}\right]\right)$ satisfaz uma identidade de grupo

Por outro lado, pela proposição 3.13, temos que $\Phi\left(G / \Phi_{p}(G)\right)$ não tem $p$-elementos, e portanto pelo lema $3.14 F\left[\frac{G}{\Phi_{p}(g)}\right]$ é semiprimo. 
Assim, pelo caso anterior temos que $F\left[\frac{G}{\Phi_{p}(G)}\right]$ é comutativo, e portanto $G / \Phi_{p}(G)$ é abeliano. Logo como $G^{\prime} \subset \Phi_{p}(G), G^{\prime}$ é um $p$-grupo finito.

Pelo lema $3.9 F[G]$ satisfaz uma identidade polinomial da forma $[x, y]^{p^{m}}=0$.

- 3- $N$ é nil mas não é nilpotente: Pela proposição 2.1 podemos supor que $U(F[G])$ satisfaz a identidade $\omega\left(x_{1}, x_{2}\right)=1$ em duas variáveis.

Seja $\mathcal{A}=F\left\langle x_{1}, x_{2}\right\rangle[[t]]$ e seja $\mathcal{F}$ o grupo livre de posto 2 .

Pela proposição 3.7 sabemos que $1+x_{1} t$ e $1+x_{2} t$, geram um grupo livre em $U(\mathcal{A})$. Temos assim:

$$
1 \neq \omega\left(1+x_{1}, 1+x_{2}\right)=1+\sum_{i=1}^{\infty} t^{i} p_{i}\left(x_{1}, x_{2}\right)
$$

onde os $p_{i}$ 's são polinômios homogêneos de grau $i$ em $F\left\langle x_{1}, x_{2}\right\rangle$. Assim podemos concluir que

$$
\sum_{i=1}^{\infty} t^{i} p_{i}\left(x_{1}, x_{2}\right) \neq 0
$$

Logo existe $p_{i_{0}}$ tal que $p_{i_{0}}\left(x_{1}, x_{2}\right) \neq 0$, e portanto $p_{i_{0}}$ é não trivial.

Afirmamos que $N(F[G])$ satisfaz $p_{i_{0}}=0$.

Sejam $r_{1}, r_{2} \in N(F[G])$. Sabemos que os elementos $1+r_{i} \lambda$ são inversíveis em $F[G]$ para todo $\lambda \in F$ com inverso

$$
1-r_{i} \lambda+r_{i}^{2} \lambda^{2}-\cdots+(-1)^{d_{i}-1} r_{i}^{d_{i}-1} \lambda^{d_{i}-1},
$$

onde $d_{i}$ é o menor inteiro tal que $r_{i}{ }^{d_{i}}=0$.

Logo $\left(1+r_{1} \lambda\right),\left(1+r_{2} \lambda\right)$ satisfazem a identidade $\omega=1$ Assim

$$
\omega\left(1+\lambda r_{1}, 1+\lambda r_{2}\right)=1,
$$

isto é,

$$
\sum_{i=1}^{\infty} \lambda^{i} p_{i}\left(r_{1}, r_{2}\right)=0
$$

Vale notar que se $\omega\left(x_{1}, x_{2}\right)$ é da forma $x_{1}{ }^{s_{1}} \cdots x_{i_{k}}{ }^{s_{k}}$, então o polinômio homogêneo de grau maior que $k$ tem em todos seus monômios um $x_{1}{ }^{2}$ ou um $x_{2}{ }^{2}$, (ou seja em alguns monômios $x_{1}^{2}$, e em outros $x_{2}^{2}$ ). Da mesma forma o polinômio homogêneo de grau 
maior que $k d_{3}$ onde $d_{3}$ é o máximo entre $d_{1}$ e $d_{2}$ tem em todos os monômios ou um $x_{1}^{d_{3}+1}$ ou um $x_{2}^{d_{3}+1}$. Usando o fato que $r_{1}{ }^{d_{3}}=r_{2}{ }^{d_{3}}=0$ e tomando $d=k d_{3}$ temos:

$$
\sum_{i=1}^{\infty} \lambda^{i} p_{i}\left(r_{1}, r_{2}\right)=\sum_{i=1}^{d} \lambda^{i} p_{i}\left(r_{1}, r_{2}\right)
$$

Em outras palavras se o nosso $i_{0}>d$ então $p_{i_{0}}\left(r_{1}, r_{2}\right)=0$. Vamos mostrar agora que $p_{i_{0}}\left(r_{1}, r_{2}\right)=0$ se $i_{0} \leq d$ :

Como $F$ é infinito tomemos $d+1$ elementos distintos em $F, \lambda_{1}, \ldots \lambda_{d+1}$ e observemos que vale a igualdade:

$$
\left(\begin{array}{llll}
1 & \lambda_{1} & \cdots & \lambda_{1}^{d} \\
1 & \lambda_{2} & \cdots & \lambda_{2}^{d} \\
\vdots & \vdots & & \vdots \\
1 & \lambda_{d+1} & \cdots & \lambda_{d+1}^{d}
\end{array}\right)\left(\begin{array}{c}
0 \\
p_{1}\left(r_{1}, r_{2}\right) \\
\vdots \\
p_{d}\left(r_{1}, r_{2}\right)
\end{array}\right)=0
$$

Pelo determinante de Vandermonde a matriz

$$
\left(\begin{array}{llll}
1 & \lambda_{1} & \cdots & \lambda_{1}^{d} \\
1 & \lambda_{2} & \cdots & \lambda_{2}^{d} \\
\vdots & \vdots & & \vdots \\
1 & \lambda_{d+1} & \cdots & \lambda_{d+1}^{d}
\end{array}\right)
$$

é inversível e portanto:

$$
p_{i}\left(r_{1}, r_{2}\right)=0
$$

para todo $i \leq d$.

Logo, para todos $r_{1}, r_{2} \in N(F[G]), p_{i_{0}}\left(r_{1}, r_{2}\right)=0$. Assim concluimos que $N(F[G])$ satisfaz uma identidade polinomial.

Usando o teorema 1.29 temos que $N(F[G])$ satisfaz uma identidade multilinear

$$
\sum_{\sigma \in S_{i_{0}}} \alpha_{\sigma} x_{\sigma(1)} \cdots x_{\sigma\left(i_{0}\right)}=0
$$

Com $\alpha_{\sigma} \in F$, para todo $\sigma$ e pelo menos um $\alpha_{\sigma^{\prime}}$ diferente de 0 .

Como $N(F[G])$ é ideal temos que $F[G]$ satisfaz a identidade polinomial multilinear generalizada:

$$
\sum_{\sigma \in S_{i_{0}}} \alpha_{\sigma} a_{1} x_{\sigma(1)} \cdots a_{i_{0}} x_{\sigma\left(i_{0}\right)}
$$


Com,

$$
a_{1} a_{2} \cdots a_{i_{0}} \neq 0,
$$

(tais $a_{i}^{\prime}$ s existem devido ao fato de $N(F[G])$ ser não nilpotente.) Segue daí que se tomarmos $x_{1}=x_{2}=\cdots x_{i_{0}}=1$,

$$
\alpha_{\sigma^{\prime}} a_{1} x_{\sigma^{\prime}(1)} \cdots a_{i_{0}} x_{\sigma^{\prime}\left(i_{0}\right)}=\alpha_{\sigma^{\prime}} a_{1} \cdots a_{i_{0}} \neq 0
$$

Logo, temos que $F[G]$ satisfaz uma identidade polinomial não degenerada.

Usando o lema 3.19 temos que $[G: \Phi]<\infty$ e $\left|\Phi^{\prime}\right|<\infty$. Vamos mostrar agora que $\Phi^{\prime}$ é p-grupo.

Pela proposição $3.12 \Phi$ é localmente finito.

Logo pelo teorema 1.5, podemos concluir que $G$ é localmente finito. Afirmamos que $\Phi^{\prime}$ é $p$-grupo:

Seja $\alpha \in \Phi^{\prime}$. O elemento $\alpha$ será da forma $\left(h_{1}, h_{2}\right) \cdots\left(h_{2 n-1}, h_{2 n}\right)$, com $h_{i} \in \Phi$. Seja $H=\left\langle h_{1}, \ldots h_{2 n}\right\rangle$. Como $\Phi$ é localmente finito $H$ será um subgrupo finito de $\Phi$. Sabemos que $U(F[H])$ satisfaz uma identidade de grupo. Assim pelo lema 3.8 $H^{\prime}$ é $p$-grupo finito, e como $\alpha \in H^{\prime}$ temos que $o(\alpha)$ é uma potência de $p$. Portanto podemos concluir que $\Phi^{\prime}$ é $p$-grupo.

Agora pelo lema 3.16 concluimos que $F[G]$ satisfaz uma identidade polinomial.

Isto termina a demonstração do teorema.

Deste teorema segue

Corolário 3.20. Sejam $F$ um corpo infinito, e $G$ um grupo de torção tal que $U(F[G])$ satisfaz uma identidade de grupo. Então $G$ é localmente finito.

\section{Demonstração:}

Pela proposição 3.12 temos que $\Phi(G)$ é localmente finito.

Pelo Teorema que acabamos de demonstrar temos que $F[G]$ satisfaz uma identidade polinomial. Usando o lema 3.18 temos que $[G: \Phi(G)]<\infty$.

Assim pelo teorema 1.5, teremos que $G$ é localmente finito.

Com o corolário acima poderemos remover a hipótese de $G$ ser finito e supor apenas $G$ de torção no lema 3.8. Enunciamos:

Lema 3.21. Sejam $G$ um grupo de torção e $F$ um corpo infinito de característica $p>0$. Se $U(F[G])$ satisfaz uma identidade de grupo, então $G^{\prime}$ é p-grupo. Se F é um corpo de característica 0 , então $G$ será um grupo abeliano. 


\section{DemonstraçÃo: Caso $p>0$}

Queremos mostrar que todo elemento de $G^{\prime}$ é um $p$-elemento de $G$.

Seja $h \in G^{\prime}, h=\left(h_{1}, h_{2}\right) \cdots\left(h_{2 n-1}, h_{2 n}\right)$ e seja $H$ o subgrupo, gerado pelos $h_{i}$ 's:

$$
H=\left\langle h_{1}, \ldots h_{2 n}\right\rangle
$$

Usando a proposição acima $G$ será localmente finito, e portanto $H$ será finito. Pelo lema 3.8, temos que $H^{\prime}$ é $p$-grupo.

Como $h \in H^{\prime}$ temos que $h$ é um $p$-elemento, e portanto $G^{\prime}$ será $p$-grupo.

Caso $p=0$

Queremos mostrar que para todos $x$ e $y$ em $G, x y=y x$. Tomando $H=\langle x, y\rangle$, temos que $H$ é finito, e portanto pelo lema $3.8, H$ será abeliano. Logo temos $x y=y x$.

Existem álgebras de grupo onde o corpo $F$ é infinito, o grupo $G$ é de torção, $F[G]$ satisfaz uma identidade polinomial mas $U(F[G])$ não satisfaz nenhuma identidade de grupo. Ou seja não vale a recíproca do nosso teorema.

\section{Por exemplo:}

Seja $F=\mathbb{Q}$, o corpo dos números racionais. $G=S_{3}$. O anel de grupo $\mathbb{Q}\left[S_{3}\right]$ é semiprimo visto que $\mathbb{Q}$ tem característica 0 . Como vimos se $U\left(\mathbb{Q}\left[S_{3}\right]\right)$ satisfazesse uma identidade de grupo $\mathbb{Q}\left[S_{3}\right]$ seria comutativo. $\operatorname{Logo} U\left(\mathbb{Q}\left[S_{3}\right]\right)$ não satisfaz nenhuma identidade de grupo.

Por outro lado, tomando a representação regular de $\mathbb{Q}\left[S_{3}\right]$, vemos que existe um isomorfismo entre $\mathbb{Q}\left[S_{3}\right]$ e um subgrupo $H$ de $M_{6}(\mathbb{Q})$. Pelo Teorema de AmitsurLevitzky, (ver teorema1.31), temos que $H$, e conseqüentemente $\mathbb{Q}\left[S_{3}\right]$, satisfazem a identidade polinomial standard de grau 12.

O próximo resultado, nos dá uma recíproca, no caso em que $G$ é grupo nilpotente, e $F[G]$ satisfaz uma identidade polonomial muito particular, relacionada com a definição abaixo:

Definição 3.22. Dizemos que um anel é Lie-n-Engel se para todo $x, y$ no anel vale a identidade $[x, \underbrace{y, \ldots, y}_{n \text { vezes }}]=0$.

Antes de enunciar, e demonstrar, o teorema, vamos enunciar um teorema devido a Sehgal:

Teorema 3.23. Seja $F$ um corpo de característica $p \geq 0$. Então, nestas condições $F[G]$ é Lie n-Engel, se e somente se: 
1. G é nilpotente e contém um subgrupo normal p-abeliano $A$, com $G / A, p$-grupo finito se $p>0$;

2. Gé abeliano se $p=0$.

DemonstraÇÃo: ver pag 155, teorema 6.1 do capítulo 5 de [Seh78].

Teorema 3.24. Seja $F$ um corpo infinito de característica $p \geq 0$ e seja $G$ um grupo nilpotente de torção. São equivalentes:

1. $U(F[G])$ satisfaz uma identidade de grupo;

2. $F[G]$ é Lie-n-Engel;

3. $U(F[G])$ satisfaz a identidade de grupo $\left(u^{p^{m}}, v\right)=1$.

DEMONSTRAÇÃO:

$(1 \Longrightarrow 2)$ :

Como $U[F(G)]$ satisfaz uma identidade de grupo, com as hipóteses acima e com o teorema 3.1 podemos concluir que $F[G]$ satisfaz uma identidade polinomial.

Se $F$ tem característica 0 , pelo lema 3.21, teremos que $F[G]$ é comutativo e portanto será Lie n-Engel.

No caso $p>0$ mostraremos que $\Phi=\Phi(G)$ é um subgrupo normal p-abeliano, e que $\frac{G}{\Phi(G)}$ é um p-grupo finito. E assim pelo teorema 3.23 , poderemos concluir que $G$ é Lie n-Engel.

Como $U(F[\Phi])$ satisfaz uma identidade de grupo, temos por 3.21 que $\Phi^{\prime}$ é p-grupo. Da proposição 3.18 segue que $\Phi^{\prime}$ é finito. Portanto $\Phi$ é $p$-abeliano.

De 3.18 também concluimos que $[G: \Phi]<\infty$, basta mostrar que $\frac{G}{\Phi}$ é $p$-grupo.

Sejam $P$ o conjunto dos $p$-elementos, e $Q$ o conjunto dos $p^{\prime}$-elementos. Como $G$ é nilpotente $P$ e $Q$ são subgrupos de $G$ e $G=P \times Q$. Do lema 3.21 temos que $G^{\prime}$ é p-grupo e portanto $G^{\prime} \leq P$. Donde concluimos que $Q$ é central e portanto é subgrupo (normal) de $\Phi$. Assim temos

$$
\frac{G}{\Phi} \cong \frac{G / Q}{\Phi / Q} \cong \frac{P}{\Phi / Q}
$$

Logo $\frac{G}{\Phi}$ é $p$-grupo.

Assim pelo teorema 3.23, concluimos que $F[G]$ é Lie-n-Engel. 
$(2 \Longrightarrow 3)$ : O caso $p=0$ segue diretamente de 3.23 , portanto vamos supor $p>0$.

Se $F[G]$ é Lie-n-Engel, então $[x, \underbrace{y, \ldots, y}_{n}]=0$

Fazendo $[x, y, \ldots, y]=\sum_{i=0}^{n}\left(\begin{array}{c}n \\ i\end{array}\right)(-1)^{i} y^{i} x y^{n-i}$ e tomando $m$ tal que $p^{m}>n$ teremos:

$$
[x, \underbrace{y, \ldots, y}_{p^{m}}]=0
$$

e portanto

$$
\sum_{i=0}^{p^{m}}\left(\begin{array}{c}
p^{m} \\
i
\end{array}\right)(-1)^{i} y^{i} x y^{p^{m}-i}=0
$$

Como $p$ divide $\left(\begin{array}{c}p^{m} \\ i\end{array}\right)$ para $1 \leq i \leq p^{m}-1$, temos

$$
x y^{p^{m}}-y^{p^{m}} x=0 .
$$

Em particular para $u, v \in U(F[G])$ temos

$$
\begin{gathered}
v u^{p^{m}}-u^{p^{m}} v=0 \Longrightarrow \\
v u^{p^{m}}=u^{p^{m}} v \Longrightarrow u^{-p^{m}} v^{-1} u^{p^{m}} v=1
\end{gathered}
$$

e portanto para todos $u, v \in U(F[G])$ vale $\left(u^{p^{m}}, v\right)=1$.

Como $(3 \Longrightarrow 1)$ é trivial temos a tese.

Para encerrar o capítulo, vale citar o fato que, recentemente C.H.Liu, demonstrou o teorema 3.1, sem a hipótese de $F$ ser infinito. Tal demonstração será parte integrante de sua tese de doutorado. 


\section{Capítulo 4}

\section{O caso modular-uma condição necessária e suficiente}

\subsection{Introdução:}

No capítulo 2 vimos algumas propriedades do anel de grupo $F[G]$ quando $U(F[G])$ satisfaz uma identidade de grupo, e obtivemos resultados importantes no caso em que o anel é semi primo. No capítulo 3 vimos que se $G$ é grupo de torção, $F$ é um corpo infinito, e $U(F[G])$ satisfaz uma identidade de grupo, então $F[G]$ satisfaz uma identidade polinomial.

Neste capítulo vamos caracterizar os grupos de torção para os quais $U(F[G])$ satisfaz a uma identidade de grupo. Vamos provar:

Teorema 4.1 (Passman, [Pas97]). Seja $U=U(F[G])$ o grupo de unidades da álgebra de grupo de um grupo de torção $G$ sobre o corpo infinito $F$ de característica $p>0$. São equivalentes:

i) U satisfaz uma identidade de grupo.

ii) G tem um subgrupo normal p-abeliano de indice finito e $G^{\prime}$ é um p-grupo de período limitado.

iii) $U$ satisfaz $(x, y)^{p^{k}}=1$ para algum $k>0$.

Claramente (iii) $\Rightarrow$ (i) é trivial.

Dedicaremos as próximas seções para demonstrar (i) $\Rightarrow$ (ii) e (ii) $\Rightarrow$ (iii). 


\subsection{A implicação (i) $\Rightarrow$ (ii)}

Nesta seção, demonstraremos basicamente que se $F$ é um corpo infinito de característica $p>0$ e $G$ é um grupo de torção que satisfaz uma identidade de grupo, então $G^{\prime}$ tem expoente limitado. As outras afirmações da tese seguem como corolário do fato que $F[G]$ satisfaz uma identidade polinomial.

Inicialmente vamos estender a proposição 1.27, com algumas restrições sobre o corpo $F$. Para isto, usaremos que com as hipóteses assumidas, o grupo $G$ é localmente finito.

Lema 4.2. Sejam $F$ um corpo infinito com caracteristica $p>0$ e $G$ um grupo de torção. Suponha que $U(F[G])$ satisfaça a identidade de grupo $\omega=1$. Se $H$ é qualquer subgrupo de $G$ ou se $\frac{G}{N}$ é qualquer imagem homomórfica de G, então $U(F[H])$ e $U\left(F\left[\frac{G}{N}\right]\right)$ também satisfazem $\omega=1$.

\section{DEMONSTRAÇÃO:}

O resultado para $H \leq G$, é trivial visto que $U(F[H]) \subseteq U(F[G])$.

Para demonstrar a afirmação para $\frac{G}{N}$ consideraremos vários casos:

$N$ é um $p$-grupo finito:

Feito na proposição 1.27 .

$N$ é um $p^{\prime}$-grupo finito:

Seja $\hat{N}=\sum_{g \in N} g, n=|N|$. Tomando $e=\frac{\hat{N}}{n}$ temos que $e$ é idempotente central, pois como $N$ é normal,

$$
\begin{gathered}
g^{-1} \frac{\hat{N}}{n} g= \\
g^{-1} \frac{\left(g^{-1}+\cdots+g_{n}\right)}{n} g= \\
\frac{g^{-1} g_{1} g+g^{-1} g_{2} g+\cdots+g^{-1} g_{n} g}{n}=\frac{\hat{N}}{n}
\end{gathered}
$$

para todo $g$ em $G$. 
Portanto

$$
F[G]=e \cdot F[G] \oplus(1-e) \cdot F[G] .
$$

Logo $U(e F[G])$ satisfaz $\omega=1$. Além disso, como $e$ é central temos,

$$
\begin{aligned}
\varphi: e F[G] & \longrightarrow F\left[\frac{G}{N}\right] \\
e \cdot \sum_{g \in G} \alpha_{g} g & \longrightarrow \sum_{g \in G} \alpha_{g} g N
\end{aligned}
$$

está bem definida e é isomorfismo.

Logo $U\left(F\left[\frac{G}{N}\right]\right)$ satisfaz $\omega=1$.

$N$ é finito:

Pelo lema 3.21, $G^{\prime}$ é p-grupo.

Se $N<G$, então $N^{\prime}<G^{\prime}$. Isto implica que se $P$ é um $p$ subgrupo de Sylow de $N$, então $N^{\prime}<P$, e portanto $P \triangleleft N$. Como $P$ é $p$ subgrupo de Sylow normal de $N$ e $N$ é finito, pelo segundo teorema de Sylow, $P$ é característico em $N$ e portanto $P \triangleleft G$. Assim podemos escrever:

$$
\frac{G}{N} \cong \frac{G / P}{N / P}
$$

Usando o primeiro caso concluimos que $U\left(F\left[\frac{G}{P}\right]\right)$ satisfaz uma identidade de grupo. ( $P$ é $p$-grupo) Como $P$ é $p$-Sylow, temos que $\frac{N}{P}$ é $p^{\prime}$-subgrupo de $\frac{G}{P}$.

Pelo caso 2, $U\left(F\left[\frac{G}{N}\right]\right)=U\left(F\left[\frac{G / P}{N / P}\right]\right)$ satisfaz $\omega=1$.

$N$ é um grupo qualquer:

Sejam $\overline{u_{1}}, \ldots, \overline{u_{n}} \in F\left[\frac{G}{N}\right]$. Queremos mostrar que:

$$
\omega\left(\overline{u_{1}}, \ldots, \overline{u_{n}}\right)=1 \text {, }
$$

para todos $\overline{u_{1}}, \ldots, \overline{u_{n}}$ pertencentes a $F[G / N]$.

Tomemos $u_{1}, \ldots, u_{n}$ e $v_{1}, \ldots, v_{n}$ tais que $\pi\left(u_{i}\right)=\bar{u}_{i}$, e $\pi\left(v_{i}\right)=\bar{u}_{i}{ }^{-1}$, onde $\pi$ é o epimorfismo canônico.

Denotaremos por $\operatorname{supp}\left(u_{i}\right)$ o suporte de cada $u_{i}$ em $F[G]$.

Seja $L=\left\langle\operatorname{supp}\left(u_{i}\right), \operatorname{supp}\left(v_{i}\right) \mid 1 \leq i \leq n\right\rangle$. 
Como $G$ é localmente finito, $L$ é finito. Seja $M=L \cap N$. Podemos afirmar, com um pequeno abuso de linguagem, que os $\bar{u}_{i}$ 's, e $\bar{u}_{i}{ }^{-1}$ 's, pertencem a $U\left(F\left[\frac{L}{M}\right]\right)$. Como $M$ é finito, segue do caso anterior que,

$$
\omega\left(\overline{u_{1}}, \ldots, \overline{u_{n}}\right)=1,
$$

Para todos $\overline{u_{1}}, \ldots, \overline{u_{n}}$ em $U\left(F\left[\frac{G}{N}\right]\right)$. E assim concluimos nossa demonstração.

Lema 4.3. Seja $G$ um grupo e seja $A$ um subgrupo abeliano normal de $G$. Suponha que $\frac{G}{A}$ é cíclico de ordem finita $q$. Se $G=\langle A, t\rangle$, então $G^{\prime}=(A, t)=\{(a, t) \mid a \in A\}$. Além disso $G \cap C_{G}(t)$ tem período dividindo $q$

\section{DEMONSTRAÇÃO:}

Primeiramente vamos demonstrar que $(A, t)$ é subgrupo de $G$.

$(A, t)$ é fechado para inversos, isto é

$$
\left(a^{-1} t^{-1} a t\right)^{-1} \in(A, t) .
$$

Como $A$ é abeliano e $A \triangleleft G$, temos

$$
\left(a^{-1} t^{-1} a t\right)^{-1}=t^{-1} a^{-1} t a=a t^{-1} a^{-1} t=\left(a^{-1}, t\right) \in(A, t) .
$$

$(A, t)$ é fechado para o produto, isto é

$$
\begin{gathered}
\left(a_{1}{ }^{-1} t^{-1} a_{1} t\right)\left(a_{2}{ }^{-1} t^{-1} a_{2} t\right) \in(A, t) . \\
a_{1}{ }^{-1} \underbrace{t^{-1} a_{1} t}_{\in A} a_{2}{ }^{-1} t^{-1} a_{2} t=a_{1}{ }^{-1} a_{2}^{-1} t^{-1} a_{1} t t^{-1} a_{2} t=\left(a_{1} a_{2}, t\right) \in(A, t)
\end{gathered}
$$

Portanto $(A, t)$ é grupo. Além disso, como $A \triangleleft G$ e $A$ é abeliano, segue que $(A, t) \leq A$.

Vamos agora mostrar que $(A, t) \triangleleft G$.

Como $A$ é abeliano e $(A, t)$ é subgrupo de $A$ segue que $(A, t) \triangleleft A$. Assim do fato que $G=\langle A, t\rangle$, basta mostrar que $t$ normaliza $(A, t)$, isto é

$$
\begin{gathered}
t^{-1}(A, t) t \leq(A, t) . \\
t^{-1} \cdot a_{1}^{-1} t^{-1} a_{1} t \cdot t=t^{-1} a_{1}{ }^{-1} t t^{-1} t^{-1} a_{1} t^{2}= \\
\underbrace{t^{-1} a_{1}^{-1} t}_{a^{\prime-1}} t^{-1} \underbrace{t^{-1} a_{1} t}_{a^{\prime}} t=a^{\prime-1} t^{-1} a^{\prime} t \in(A, t) .
\end{gathered}
$$


Assim concluimos que t normaliza $(A, t)$, e portanto, $(A, t) \triangleleft G$.

Desta forma, podemos escrever:

$$
\frac{G}{A}=\frac{G /(A, t)}{A /(A, t)}
$$

Claramente temos que $(A, t) \leq G^{\prime}$. Para conseguir $G^{\prime} \leq(A, t)$ mostraremos que $\frac{G}{(A, t)}$ é abeliano. Usaremos para isso o fato que se o quociente de um grupo por um subgrupo central é cíclico, então este grupo é abeliano.

Basta provar então que $A /(A, t)$ é subgrupo central de $G /(A, t)$, ou equivalentemente, mostrar que para todo $g \in G$ e para todo $a \in A$ temos $g^{-1} a^{-1} g a \in(A, t)$. temos

Como $A \triangleleft G$ temos que $A \cdot\langle t\rangle$ será subgrupo de $G$. Como $A$ e $t$ estão em $A \cdot\langle t\rangle$,

$$
G=A \cdot\langle t\rangle
$$

Afirmamos que dado um $g=a t^{n} \in G$ e um $b \in A$ temos:

$$
g^{-1} b^{-1} g b \in(A, t)
$$

Como $A$ é abeliano, e normal em $G$, segue que:

$$
g^{-1} b^{-1} g b=t^{-n} \underbrace{a^{-1} b^{-1} a}_{=b^{-1}} t^{n} b=b t^{-n} b^{-1} t^{n}
$$

Logo basta provar que para todo $n \in \mathbb{Z}$ e para todo $a \in A$ temos

$$
a^{-1} t^{-n} a t^{n} \in(A, t):
$$

$n=0$, Trivial.

$n>0$. Usaremos indução em $n$.

Para $n=1$ não há o que fazer. Vamos supor que valha para $n-1$, ou seja: $a^{-1} t^{-(n-1)} a t^{n-1} \in(A, t)$, para todo $a \in A$

Assim temos:

$$
a^{-1} t^{-n} a t^{n}=a^{-1} t^{-1} \underbrace{a t t^{-1} a^{-1}}_{=1} t^{-(n-1)} a t^{n-1} t=
$$




$$
\left(a^{-1} t^{-1} a t\right) \cdot t^{-1} \cdot\left(a^{-1} t^{-(n-1)} a t^{n-1}\right) \cdot t
$$

Como $(A, t) \triangleleft G$ segue da hipótese de indução que $t^{-1} a^{-1} t^{-(n-1)} a t^{n-1} t$ pertence a $(A, t)$, e assim $a^{-1} t^{-n} a t^{n} \in(A, t)$. Logo concluimos o caso em que $n>0$. Vamos agora considerar o caso $n<0$.

Para facilitar vamos fazer $m=-n>0$, e provar

$$
a^{-1} t^{m} a t^{-m} \in(A, t) \text {. }
$$

Temos

$$
\begin{gathered}
a^{-1} t^{m} a t^{-m}=t^{m} a t^{-m} a^{-1}= \\
t^{m} a t^{-m} t^{-m} t^{m} a^{-1} t^{-m} t^{m}=\left(t^{m} a t^{-m}\right) t^{-m}\left(t^{m} a^{-1} t^{-m}\right) t^{m}= \\
a^{-1} t^{-m} a^{\prime} t^{m},
\end{gathered}
$$

onde $a^{\prime}=t^{m} a^{-1} t^{-m} \in A$ devido ao fato de $A \triangleleft G$.

Assim concluimos que $A /(A, t)$ é subgrupo central de $G /(A, t)$, e por consequência que $(A, t)=G^{\prime}$.

Mostraremos agora que o período de $\left(G^{\prime} \cap C_{G}(t)\right)$ divide $q$.

Seja $b \in G^{\prime} \cap C_{G}(t)$. Usando o resultado anterior $b$ será da forma $a^{-1} t^{-1} a t$ para algum $a \in A$. Além disso $b t=t b$ o que implica $t\left(a^{-1} t^{-1} a t\right)=a^{-1} t^{-1} a t^{2}$.

Vamos provar que $b^{n}=a^{-1} t^{-n} a t^{n}$ para todo $n$, positivo.

A afirmação é verdadeira para $n=1$.

Suponha $b^{n-1}=a^{-1} t^{-(n-1)} a t^{n-1}$. Certamente $t$ comuta com $b^{n-1}$ e portanto

$$
\begin{gathered}
b^{n}=a^{-1} t^{-1} a t a^{-1} t^{-(n-1)} a t^{n-1}=a^{-1} t^{-1} t^{-(n-1)} a t^{n-1} t= \\
a^{-1} t^{-n} a t^{n} .
\end{gathered}
$$

Assim, do fato que $G / A$ é cíclico de período $q$, temos que $t^{q} \in A$. Logo, como $A$ é abeliano.

$$
b^{q}=a^{-1} t^{-q} a t^{q}=a^{-1} a t^{-q} t^{q}=1 .
$$

Portanto o período de $\left(G^{\prime} \cap C_{G}(t)\right)$ divide $q$.

Vamos agora construir uma função que exercerá um papel fundamental na demonstração dos próximos lemas desta seção.

Suponha $G$ um grupo, $A$ um subgrupo normal abeliano de $G$ e $t$ um elemento de $G$ com ordem prima $q$, de tal forma que

$$
G=A \rtimes\langle t\rangle \text {. }
$$


Definição 4.4. A função traço é a aplicação de $F[A]$ em $F[A]$, dada por

$$
\operatorname{Tr}(\sigma)=\sigma+t^{-1} \sigma t+\cdots t^{-(q-1)} \sigma t^{q-1},
$$

para todo $\sigma$ pertencente a $F[A]$.

Como $A$ é subgrupo normal de $G$ temos que a imagem da função traço está contida em $F[A]$. Além disso, podemos verificar facilmente que:

$$
\begin{gathered}
\operatorname{Tr}\left(\sigma_{1}+\sigma_{2}\right)=\operatorname{Tr}\left(\sigma_{1}\right)+\operatorname{Tr}\left(\sigma_{2}\right), \\
\operatorname{Tr}(\lambda \sigma)=\lambda \operatorname{Tr}(\sigma),
\end{gathered}
$$

para todo $\lambda$ pertencente a $F$, e $\sigma, \sigma_{1}, \sigma_{2}$ pertencentes a $F[A]$.

Vamos agora enumerar outras propriedades desta função:

Proposição 4.5. Para todo $\sigma$ pertencente a $F[A]$ temos $\operatorname{Tr}(\sigma) \in Z(F[G])$

\section{DEMONSTRAÇÃO:}

Basta demonstrar que $\operatorname{Tr}(\sigma)$ comuta com os elementos de $G$.

Para todo $g \in G$, temos:

$$
\begin{aligned}
& g^{-1} \operatorname{Tr}(\sigma) g=g^{-1} \sigma g+\cdots+g^{-1} t^{-(q-1)} \sigma t^{(q-1)} g= \\
& =t^{-l} a_{1}^{-1} \sigma a_{1} t^{l}+\cdots+t^{-l} a_{1}^{-1}\left(t^{-(q-1)} \sigma t^{q-1}\right) a_{1} t^{l},
\end{aligned}
$$

pois como $G=A \rtimes\langle t\rangle$, para todo $g \in G$ existem $a_{1} \in A$ e $l \in \mathbb{N}, 0 \leq l \leq(q-1)$, tais que $g=a_{1} \cdot t^{l}$.

Mas como A é abeliano e $t^{n} \mapsto t^{n} \cdot t^{l}$ é uma bijeção de $\langle t\rangle$ em $\langle t\rangle$, segue que:

$$
\begin{gathered}
g^{-1} \operatorname{Tr}(\sigma) g= \\
t^{-l} \sigma t^{l}+\cdots+t^{-l} t^{-(q-1)} \sigma t^{q-1} t^{l}=\operatorname{Tr}(\sigma) .
\end{gathered}
$$

E portanto $g \cdot \operatorname{Tr}(\sigma)=\operatorname{Tr}(\sigma) \cdot g$, logo $\operatorname{Tr}(\sigma) \in Z(F[G])$.

Seja $G$ um grupo e $x$ e $y$ elementos de $G$. Vamos notar por $x^{y}$ o elemento $y^{-1} x y$.

Proposição 4.6. Para todo o pertencente a $F[A]$ temos

$$
\operatorname{Tr}(\sigma)^{p}=\operatorname{Tr}\left(\sigma^{p}\right) .
$$




\section{DEMONSTRAÇÃO:}

Como $A$ é abeliano e normal em $G t^{-j} \sigma t^{j}$ comuta com $t^{-i} \sigma t^{i}$ para todo $i$ e $j$. Assim:

$$
\begin{gathered}
\operatorname{Tr}(\sigma)^{p}=\left(\sigma+\sigma^{t}+\ldots+\sigma^{t^{q-1}}\right)^{p}= \\
\sigma^{p}+\left(\sigma^{t}\right)^{p}+\cdots\left(\sigma^{t^{q-1}}\right)^{p} .
\end{gathered}
$$

Como

$$
\left(\sigma^{t^{i}}\right)^{p}=\left(t^{-i} \sigma t^{i}\right)^{p}=t^{-1} \sigma^{p} t^{i}=\left(\sigma^{p}\right)^{t^{i}}
$$

segue que $\operatorname{Tr}(\sigma)^{p}=\operatorname{Tr}\left(\sigma^{p}\right)$.

Proposição 4.7. Para todo $\zeta \in F[A] \cap Z(F[G])$, e para todo $\sigma \in F[A]$ temos:

$$
\operatorname{Tr}(\sigma \zeta)=\zeta \operatorname{Tr}(\sigma)
$$

Em particular, se tomarmos $\sigma=1$ teremos

$$
\operatorname{Tr}(\zeta)=\operatorname{Tr}(1) \zeta=q \zeta
$$

Ainda, $\operatorname{Tr}(\operatorname{Tr}(\sigma))=q \cdot \operatorname{Tr}(\sigma)$, para todo $\sigma$ em $F[A]$.

DEMONSTRAÇÃO:

Se $\zeta \in F[A] \cap Z(F[G])$, então,

$$
\operatorname{Tr}(\sigma \zeta)=\sigma \zeta+t^{-1} \sigma t \zeta+\ldots+t^{-(q-1)} \sigma t^{q-1} \zeta=\operatorname{Tr}(\sigma) \zeta
$$

Em particular se $\sigma=1$ temos, $\operatorname{Tr}(\zeta)=\operatorname{Tr}(\zeta \cdot 1)=\zeta \operatorname{Tr}(1)=q \zeta$.

Notaremos por $\tau$ a soma:

$$
\tau=1+t+\ldots+t^{q-1}
$$

Vale observar que para todo $i$ teremos $t^{i} \tau=\tau$ e portanto teremos $\tau^{2}=q \tau$.

Proposição 4.8. Seja $\tau$ definido como acima. Então $\tau \sigma \tau=\operatorname{Tr}(\sigma) \tau$, para todo $\sigma \in F[A]$.

DEMONSTRAÇÃO:

$$
\tau \sigma \tau=\sum_{i=0}^{q-1} \sum_{j=0}^{q-1} t^{-i} \sigma t^{j}=\sum_{i=0}^{q-1}\left(\sum_{j=0}^{q-1}\left(t^{-i} \sigma t^{i}\right) t^{j-i}\right)=\sum_{i=0}^{q-1} t^{-i} \sigma t^{i} \sum_{j=0}^{q-1} t^{j-i}=
$$


Para cada $i$ temos $\sum_{j=0}^{q-1} t^{j-i}=\tau, \log 0$

$$
\tau \sigma \tau=\left(\sum_{i=0}^{q-1} t^{-i} \sigma t^{i}\right) \tau=\operatorname{Tr}(\sigma) \tau
$$

No próximo lema iremos demonstrar que $G^{\prime}$ tem período finito para uma classe muito restrita de grupos.

Lema 4.9. Sejam $F$, um corpo infinito de característica $p>0$ e $G=\langle A, t\rangle$, onde $A$ é um subgrupo abeliano normal de $G$ e $t$ tem ordem $q, q$ um número primo. Então se $U(F[G])$ satisfaz uma identidade de grupo $G^{\prime}$ tem período finito.

\section{DEMONSTRAÇÃO:}

Dividiremos a demonstração em 2 casos:

Caso 1: $q \neq p$ :

Seja $a \in A$, definiremos $\alpha$ e $\beta$, tais que $\alpha^{2}=\beta^{2}=0$.

$$
\alpha=\tau a^{-1}\left(1-t^{-1}\right) .
$$

Temos

$$
\alpha^{2}=\tau a^{-1}\left(1-t^{-1}\right) \tau a^{-1}\left(1-t^{-1}\right)=0,
$$

pois $\left(1-t^{-1}\right) \tau=0$.

E seja $\beta$

$$
\beta=(q a-\operatorname{Tr}(a)) \tau .
$$

Como $\operatorname{Tr}(a) \in F[A] \cap Z(F[G])$, pela proposição 4.7 segue que

$$
\operatorname{Tr}(q a-\operatorname{Tr}(a))=\operatorname{Tr}(q a)-\operatorname{Tr}(\operatorname{Tr}(a))=q \operatorname{Tr}(a)-q \operatorname{Tr}(a)=0 .
$$

Assim teremos

$$
\beta^{2}=(q a-\operatorname{Tr}(a)) \underbrace{\tau(q a-\operatorname{Tr}(a)) \tau}_{\operatorname{Tr}(q \cdot a-\operatorname{Tr}(a)) \tau=0}=0 .
$$

Como $U(F[G])$ satisfaz uma identidade de grupo, segue pelo teorema 2.2 que existe um $n$ tal que se $x^{2}=y z=0$ então $y x z \cdot F[G]$ é ideal nil de expoente limitado $n$ ( $n$ dependendo somente da identidade de grupo em questão.). 
Tomando $x=\beta$ e $y=z=\alpha$ teremos que $(\alpha \beta \alpha \beta)^{n}=0$ para tal $n$. Portanto tomando um $k$ tal que $p^{k} \geq 2 n$, teremos por um lado:

$$
(\alpha \beta)^{p^{k}}=0 .
$$

Por outro lado temos:

$$
\begin{gathered}
\alpha \beta=\tau a^{-1}\left(1-t^{-1}\right)(q a-\operatorname{Tr}(a)) \tau= \\
=\tau a^{-1}\left(1-t^{-1}\right) q a \tau-\tau a^{-1}\left(1-t^{-1}\right) \operatorname{Tr}(a) \tau={ }^{1} \\
=\tau a^{-1}\left(1-t^{-1}\right) q a \tau=q \tau\left(1-a^{-1} t^{-1} a\right) \tau= \\
=q \tau\left(1-a^{-1} a^{t} t^{-1}\right) \tau=q \tau(\tau-a^{-1} a^{t} \underbrace{t^{-1} \tau}_{=\tau})= \\
=q \tau\left(1-a^{-1} a^{t}\right) \tau=q \tau^{2}-q \tau\left(a^{-1} a^{t}\right) \tau= \\
=q^{2} \tau-q \operatorname{Tr}\left(a^{-1} a^{t}\right) \tau=q\left(q-\operatorname{Tr}\left(a^{-1} a^{t}\right)\right) \tau .
\end{gathered}
$$

Seja $b=\left(a^{-1} a^{t}\right)=a^{-1} t^{-1} a t \in A$. Então

$$
\alpha \beta=q(q-\operatorname{Tr}(b)) \tau
$$

e como $q(q-\operatorname{Tr}(b))$ é central

$$
(\alpha \beta)^{p^{k}}=q^{p^{k}}(q-\operatorname{Tr}(b))^{p^{k}} \tau^{p^{k}}=q^{p^{k}}\left(q^{p^{k}}-\operatorname{Tr}(b)^{p^{k}}\right) \tau^{p^{k}} .
$$

Mas $q^{p^{k}}=q$ e $q^{p^{k}-1}=1$ e portanto

$$
(\alpha \beta)^{p^{k}}=q\left(q-\operatorname{Tr}(b)^{p^{k}}\right) \tau^{p^{k}} .
$$

Levando em conta que $\tau^{2}=q \tau$, podemos concluir que $\tau^{n}=q^{n-1} \tau$, sempre que $n \geq 2$, $\operatorname{logo} \tau^{p^{k}}=q^{p^{k}-1} \tau=\tau$, e assim

$$
(\alpha \beta)^{p^{k}}=q\left(q-\operatorname{Tr}(b)^{p^{k}}\right) \tau
$$

Por $(*)$ temos:

$$
q\left(q-\operatorname{Tr}(b)^{p^{k}}\right) \tau=0
$$

o que implica

$$
\left(q-\operatorname{Tr}(b)^{p^{k}}\right) \tau=0
$$

\footnotetext{
${ }^{1}$ o segundo termo da soma se anula, pois $\operatorname{Tr}(a)$ é central e $\left(1-t^{-1}\right) \cdot \tau=0$.
} 
Afirmamos que $q-\operatorname{Tr}(b)^{p^{k}} \in F[A]$, é igual a 0 . De fato fazendo

$$
\left(q-\operatorname{Tr}(b)^{p^{k}}\right)=\alpha_{1} a_{1}+\cdots+\alpha_{n} a_{n},
$$

$\operatorname{com} a_{1}, \ldots a_{n} \in A$ temos

$$
\begin{gathered}
\left(\alpha_{1} a_{1}+\cdots+\alpha_{n} a_{n}\right) \cdot\left(1+t+\cdots+t^{q-1}\right)= \\
\alpha_{1} a_{1}+\cdots \alpha_{n} a_{n}+\alpha_{1} a_{1} t+\cdots+\alpha_{1} a_{1} t^{q-1}+\cdots \alpha_{n} a_{n} t^{q-1}=0
\end{gathered}
$$

$\operatorname{com} a_{1}, \ldots, a_{n} \in A$, e $\alpha_{1}, \ldots, \alpha_{n} \in F$.

Como $A \cap\langle t\rangle=(1)$, se $0 \leq \beta_{1}, \beta_{2} \leq q-1$, a igualdade $a_{i_{1}} t^{\beta_{1}}=a_{i_{2}} t^{\beta_{2}}$ é equivalente a $a_{i_{1}}=a_{i_{2}}$ e $\beta_{1}=\beta_{2}$.

Daqui concluimos que $\alpha_{1}=\alpha_{2}=\cdots \alpha_{n}=0$, e portanto, $q-\operatorname{Tr}(b)^{p^{k}}=0$.

Agora pela proposição 4.6, obtemos $\operatorname{Tr}\left(b^{p^{k}}\right)=q$.

Assim, $b^{p^{k}}+t^{-1} b^{p^{k}} t+\cdots t^{-(q-1)} b^{p^{k}} t^{q-1}=q$.

Segue daí que existe um $i$ tal que

$$
t^{-i} b^{p^{k}} t^{i}=1
$$

e portanto,

$$
b^{p^{k}}=1
$$

Logo $(a, t)^{p^{k}}=1$, Para todo $a \in A$.

Assim concluimos que $(A, t)$ tem expoente limitado menor ou igual a $p^{k}$. Pelo lema 4.3 temos que $G^{\prime}=(A, t)$, e portanto $G^{\prime}$ tem expoente limitado menor ou igual a $p^{k}$.

Caso 2: $q=p$

Seja $a \in A$. Como no caso anterior definiremos $\alpha$ e $\beta$ tal que $\alpha^{2}=\beta^{2}=0$.

Seja $\alpha=\tau$

$$
\tau^{2}=p \tau=0
$$

e $\beta=a^{-1} \tau a$,

$$
\beta^{2}=\left(a^{-1} \tau a\right)^{2}=a^{-1} \tau^{2} a=0
$$

Usando raciocínio análogo ao caso anterior temos que $\beta \alpha \beta F[G]$ é ideal nil a direita de grau limitado menor ou igual a $n$, onde $n$ é um número que não depende de $a$. 
Portanto existe $k>0$ tal que

$$
(\beta \alpha)^{p^{k}}=0
$$

Observando que

$$
\beta \alpha=a^{-1} \tau a \tau=a^{-1} \operatorname{Tr}(a) \tau
$$

temos

$$
\begin{gathered}
(\beta \alpha)^{2}=a^{-1} \operatorname{Tr}(a) \tau a^{-1} \operatorname{Tr}(a) \tau= \\
a^{-1} \operatorname{Tr}(a) \tau a^{-1} \tau \operatorname{Tr}(a)=a^{-1} \operatorname{Tr}(a) \operatorname{Tr}\left(a^{-1}\right) \tau \operatorname{Tr}(a)= \\
\operatorname{Tr}(a) \operatorname{Tr}\left(a^{-1}\right) a^{-1} \operatorname{Tr}(a) \tau=\operatorname{Tr}(a) \operatorname{Tr}\left(a^{-1}\right) \beta \alpha .
\end{gathered}
$$

Se $i \geq 2$ temos, por um argumento de indução:

$$
(\beta \alpha)^{i}=\left[\operatorname{Tr}\left(a^{-1}\right) \cdot \operatorname{Tr}(a)\right]^{i-1} \cdot \beta \alpha .
$$

Usando o fato que $\operatorname{Tr}(F[A]) \subseteq Z(F[G])$ e desenvolvendo $\tau(\beta \alpha)^{p^{k}}=0$ temos

$$
\begin{gathered}
\tau(\beta \alpha)^{p^{k}}=\left[\operatorname{Tr}\left(a^{-1}\right) \operatorname{Tr}(a)\right]^{p^{k}-1} \tau \beta \alpha= \\
=\left[\operatorname{Tr}\left(a^{-1}\right) \operatorname{Tr}(a)\right]^{p^{k}-1} \operatorname{Tr}(a) \tau a^{-1} \tau= \\
=\left[\operatorname{Tr}\left(a^{-1}\right) \operatorname{Tr}(a)\right]^{p^{k}-1} \operatorname{Tr}(a) \operatorname{Tr}\left(a^{-1}\right) \tau= \\
=\left[\operatorname{Tr}\left(a^{-1}\right) \operatorname{Tr}(a)\right]^{p^{k}} \tau=0 .
\end{gathered}
$$

Como $\langle t\rangle \cap A=(1)$ e $\left[\operatorname{Tr}\left(a^{-1}\right) \cdot \operatorname{Tr}(a)\right]^{p^{k}} \in F[A]$, usando o mesmo raciocínio do primeiro caso, concluimos a partir de $\left[\operatorname{Tr}\left(a^{-1}\right) \cdot \operatorname{Tr}(a)\right]^{p^{k}} \cdot\left(1+t+\cdots t^{p-1}\right)=0$, que

$$
\left[\operatorname{Tr}\left(a^{-1}\right) \cdot \operatorname{Tr}(a)\right]^{p^{k}}=0 \text {. }
$$

Seja $b=a^{p^{k}}$. Por 4.6 temos

$$
0=\left[\operatorname{Tr}\left(a^{-1}\right) \operatorname{Tr}(a)\right]^{p^{k}}=\operatorname{Tr}\left(a^{-1}\right)^{p^{k}} \operatorname{Tr}(a)^{p^{k}}=\operatorname{Tr}\left(a^{-p^{k}}\right) \operatorname{Tr}\left(a^{p^{k}}\right)=\operatorname{Tr}\left(b^{-1}\right) \operatorname{Tr}(b) .
$$

E por 4.7

$$
\begin{aligned}
0=\operatorname{Tr}\left(b^{-1}\right) \cdot \operatorname{Tr}(b)=\operatorname{Tr}\left(b^{-1}\right. & \cdot \operatorname{Tr}(b))=\operatorname{Tr}\left(b^{-1}+b^{-1} \cdot b^{t}+\cdots+b^{-1} \cdot b^{t^{p-1}}\right)= \\
& =\sum_{i=0}^{p-1} \operatorname{Tr}\left(b^{-1} \cdot b^{t^{i}}\right) .
\end{aligned}
$$


Como

$$
\operatorname{Tr}\left(b^{-1} \cdot b^{t^{0}}\right)=\operatorname{Tr}\left(b^{-1} \cdot b\right)=\operatorname{Tr}(1)=p=0
$$

segue que

$$
\begin{gathered}
0=\sum_{i=1}^{p-1} \operatorname{Tr}\left(b^{-1} \cdot b^{t^{i}}\right)= \\
\sum_{i=1}^{p-1} \sum_{j=0}^{p-1}\left(b^{-1} \cdot b^{t^{i}}\right)^{t^{j}}= \\
\sum_{i=1, j=0}^{p-1} b^{-t^{j}} \cdot b^{t^{i+j}} .
\end{gathered}
$$

Temos então a soma de $p(p-1)$ elementos de $G$, em $F[G]$.

Esta soma só será igual a 0 se para cada $b^{-t^{j}} b^{t^{i+j}}$ existir um número $k p$ de elementos iguais a ele. Ou seja, para cada

$$
i_{0}, j_{0} \operatorname{com} i_{0} \geq 1, j_{0} \geq 0
$$

devemos ter

$$
\left|\left\{(i, j) \mid b^{-t^{j}} \cdot b^{t^{i+j}}=b^{-t^{j_{0}}} \cdot b^{t^{i_{0}+j_{0}}}\right\}\right|=k p,
$$

para algum $k \in \mathbb{N}, k$ diferente de 0 .

Logo

$$
\left|\left\{b^{-t^{j}} b^{t^{i+j}} \mid 0 \leq j \leq p-1,1 \leq i \leq p-1\right\}\right| \leq \frac{p(p-1)}{p}=p-1,
$$

em particular,

$$
\left|\left\{b^{-t^{j}} \cdot b^{t^{1+j}} \mid 0 \leq j \leq p-1\right\}\right| \leq p-1
$$

Portanto existem $j_{1}$ e $j_{2}$ distintos tais que

$$
b^{-t^{j_{1}}} b^{t^{1+j_{1}}}=b^{-t^{j_{2}}} b^{t^{1+j_{2}}}
$$

ou

$$
b^{(t-1) t^{j_{1}}}=b^{(t-1) t^{j_{2}}} .
$$

Supondo $j_{2}>j_{1}$, temos:

$$
b^{(t-1)}=b^{(t-1) \cdot t^{j_{2}-j_{1}}},
$$

que implica

$$
b^{t-1}=t^{j_{1}-j_{2}} b^{(t-1)} t^{j_{2}-j_{1}}
$$


$\operatorname{logo} b^{t-1}$ comuta com $t^{j_{2}-j_{1}}$.

Como $|\langle t\rangle|$ é primo, e $j_{2}-j_{1}$ não é múltiplo de $p$, o elemento $t^{j_{2}-j_{1}}$ gera $\langle t\rangle$. Portanto $b^{(t-1)}$ comuta com $t$. Assim $b^{-1} b^{t} \in C_{G}(t)$ o que implica que $b^{-1} b^{t} \in(A, t) \cap$ $C_{G}(t)$.

Pelo lema 4.3 temos

$$
\left(b^{-1} b^{t}\right)^{p}=1=\left(a^{-p^{k}} t^{-1} a^{p^{k}} t\right)^{p}=\left(a^{-1} t^{-1} a t\right)^{p^{k+1}} .
$$

Como tomamos $a$ arbitrário em $A$ e $G^{\prime}=(A, t)$ temos que $G^{\prime}$ tem período menor ou igual a $p^{k+1}$ e portanto $G^{\prime}$ tem período limitado.

Vamos remover agora a hipótese de $q$ ser primo:

Lema 4.10. Sejam $G=\langle A, t\rangle$ onde $A$ é um subgrupo abeliano normal e t tem ordem $q$, e $F$ um corpo infinito de característica $p>0$. Então se $U(F[G])$ satisfaz uma identidade de grupo $G^{\prime}$ tem período finito.

\section{DEMONSTRAÇÃO:}

Vamos fazer indução em $q$.

Se $q$ é primo segue pelo lema 4.9 que se $U(F[G])$ satisfaz uma identidade de grupo, então $G^{\prime}$ tem período finito.

Se $q$ não é primo, existe $\langle s\rangle$ subgrupo próprio de $\langle t\rangle$, com $\langle s\rangle \neq 1$

Seja $H=\langle A, s\rangle$. Como $H$ satisfaz a hipótese de indução $H^{\prime}$ tem período finito, e pelo lema $4.3 H^{\prime}=(A, s)$.

Tomemos $B=\left\langle H^{\prime}, s\right\rangle$

Como $H^{\prime} \triangleleft B$, temos que $B=H^{\prime} \cdot\langle s\rangle$, e portanto, todo $b \in B$, é da forma

$$
b=h k,
$$

com $h \in H^{\prime}$, e $k \in\langle s\rangle$. Vamos provar que $B$ tem período finito e que $B \triangleleft G$.

$B$ tem período finito:

Seja $b \in B, b=h k$, com $h \in H^{\prime}$ e, $k \in\langle s\rangle$.

Seja $\bar{b}=b \cdot H^{\prime}$, temos

$$
\bar{b}=\bar{h} \bar{k}=\bar{k},
$$

visto que $h \in H^{\prime}$.

Logo, como

$$
|\langle s\rangle|<|\langle t\rangle|=q<\infty,
$$


notando por $j$ a ordem de $s$ temos $b^{j} \in H^{\prime}$.

Como $H^{\prime}$ tem período finito, digamos $n$, temos

$$
\left(b^{j}\right)^{n}=b^{j n}=1
$$

para todo $b \in B$.

Logo $B$ tem período finito. (período de $B \leq j n$ ).

$B$ é normal em $G$ :

Basta mostrar que $A$ e $t$ normalizam $B$, pois $G=\langle A, t\rangle$.

\section{$A$ normaliza $B$ :}

Como $H^{\prime} \leq B$, temos $B \triangleleft H$. Portanto, como $A \leq H$, temos que $A$ normaliza $B$.

$\langle t\rangle$ normaliza $B$ :

Seja $b \in B, b=h k$ com $h \in H^{\prime}$ e $k \in\langle s\rangle$. Usando a proposição 4.3 temos que $h$ é da forma $\left(a_{\alpha}, s_{\alpha}\right)$, com $a_{\alpha} \in A$, e $s_{\alpha} \in\langle s\rangle$. De $\langle s\rangle\langle\langle t\rangle$ segue que $t$ comuta com $k$ e com $s_{\alpha}$. Temos então

$$
\begin{gathered}
t^{-1} b t=t^{-1} h k t=t^{-1} h t k= \\
=t^{-1} a_{\alpha}^{-1} s_{\alpha}^{-1} a_{\alpha} s_{\alpha} t k= \\
=t^{-1} a_{\alpha}^{-1} t s_{\alpha}^{-1} t^{-1} a_{\alpha} t s_{\alpha} k .
\end{gathered}
$$

Além disto, como $A \triangleleft G$ temos $t^{-1} a_{\alpha} t \in A$, e fazendo $a_{\beta}=t^{-1} a_{\alpha} t$ teremos,

$$
t^{-1} b t=\underbrace{a_{\beta}{ }^{-1} s_{\alpha}{ }^{-1} a_{\beta} s_{\alpha}}_{\in H^{\prime}} \underbrace{k}_{\langle s\rangle} \in B .
$$

Logo $B \triangleleft G$.

Podemos portanto considerar $\bar{G}=\frac{G}{B}=\langle\bar{A}, \bar{t}\rangle$.

Claramente $\bar{A} \triangleleft \bar{G}$ e $\bar{A}$ é abeliano. Também vale que $o(\bar{t}) \leq \frac{o(t)}{o(s)}$ e como $\langle s\rangle \neq(1)$, $o(\bar{t})<o(t)$. Pelo lema 4.2 temos que $U(F[\bar{G}])$ satisfaz a mesma identidade de grupo que $U(F[G])$. Assim podemos usar a hipótese de indução e, concluir que $\bar{G}^{\prime}$ tem período finito.

Assim, como

$$
\bar{G}^{\prime}=\left(\frac{G}{B}\right)^{\prime}=\frac{G^{\prime} \cdot B}{B} \cong \frac{G^{\prime}}{G^{\prime} \cap B},
$$


e $G^{\prime} \cap B \leq B$ tem período finito, $G^{\prime}$ tem período finito.

$$
\left(\operatorname{per}\left(G^{\prime}\right) \leq \operatorname{per}(B) \cdot \operatorname{per}\left(\bar{G}^{\prime}\right) \cdot\right)
$$

Vamos agora supor apenas que $G$ tem um subgrupo abeliano normal, de índice finito. Neste próximo caso $G$ pode ter mais geradores fora do subgrupo $A$. Aqui usaremos que $G$ é um grupo de torção.

Lema 4.11. Sejam $G$ grupo de torção e $F$ um corpo infinito de característica $p>$ 0 . Se $U(F[G])$ satisfaz uma identidade de grupo e $G$ tem um subgrupo normal $A$ abeliano, de índice finito, então $G^{\prime}$ tem período limitado.

\section{DEMONSTRAÇÃO:}

Nosso intuito nesta demonstração será construir um subgrupo $B$ normal em $G$, de período limitado, tal que $\left(\frac{G}{B}\right)^{\prime}$ tenha período finito.

Seja

$$
\left.B=\left\langle L^{\prime}\right| A \leq L \leq G, \frac{L}{A} \text { é cíclico }\right\rangle .
$$

Vamos inicialmente mostrar que $B$ tem período finito:

Como $[G: A]<\infty$, pelo teorema da correspondência, existirá uma relação biunívoca entre os conjuntos $\{L \mid A \leq L \leq G\}$, e $\left\{\frac{L}{A} \mid \frac{L}{A} \leq \frac{G}{A}\right\}$, e portanto $\{L \mid A \leq$ $L \leq G\}$ será finito.

Como $\frac{L}{A}$ é cíclico, temos $L=\langle A, t\rangle$, com $t \in G$. Como $o(t)$ é finita, pelo lema 4.10, $L^{\prime}$ tem período finito, e pelo lema $4.3, L^{\prime} \leq A$, pois $L^{\prime}=(A, t)$ e $A$ é normal em $G$. Logo $B \leq A$, e portanto $B$ é abeliano.

Ora $B$ é um grupo gerado por um número finito de grupos de período finito que é abeliano. $\operatorname{Logo} B$ terá período finito.

Vamos mostrar agora que $B$ é normal em $G$.

Se $A \leq L \leq G$, para todo $g \in G$, temos

$$
g^{-1} A g \leq g^{-1} L g \leq G
$$

Como $A \triangleleft G$, para todo $g \in G, A=g^{-1} A g$, e portanto,

$$
\frac{g^{-1} L g}{A} \cong \frac{L}{A} \text {. }
$$

Logo $\frac{g^{-1} L g}{A}$ será cíclico, e portanto $\left(g^{-1} L g\right)^{\prime}$ estará contido em $B$. 
Seja $b \in B, b=h_{1} \cdots h_{n}, \operatorname{com} h_{i} \in L_{i}{ }^{\prime} \operatorname{com} \frac{L_{i}}{A}$ cíclico. Para todo $g \in G$ temos

$$
g^{-1} b g=g^{-1} h_{1} g \cdots g^{-1} h_{n} g
$$

Como $g^{-1} h_{i} g \in\left(g^{-1} L_{i} g\right)^{\prime}$ para todo $i$, e $\left(g^{-1} L_{i} g\right)^{\prime} \leq B$ sempre que $L_{i}{ }^{\prime} \leq B$ temos que $g^{-1} h_{i} g \in B$ para todo $i$ e portanto $g^{-1} b g \in B$, para todo $g \in G$.

Logo $B \triangleleft G$.

Vamos agora demonstrar que $\left(\frac{G}{B}\right)^{\prime}$ é finito.

Se observarmos que para todo $g \in G$, o grupo $\frac{\langle A, g\rangle}{A}$ é cíclico e que pelo lema 4.3, $\langle A, g\rangle^{\prime}=(A, g) \leq B$, temos que $a^{-1} g^{-1} a g \in B$ para todo $g \in G$, e para todo $a \in A$

Portanto temos

$$
a g B=g a B,
$$

para todo $a \in A$ e para todo $g \in G$ donde concluimos que $\frac{A}{B}$ é central em $\frac{G}{B}$.

Usando o fato que $\left[\frac{G}{B}: \frac{A}{B}\right]=[G: A]<\infty$, do lema 1.3 temos que $\left(\frac{G}{B}\right)^{\prime}$ é finito.

Como $L \leq G$, temos que $L^{\prime} \leq G^{\prime}$ e portanto $B \leq G^{\prime}$ e assim

$$
\left(\frac{G}{B}\right)^{\prime}=\frac{G^{\prime} \cdot B}{B}=\frac{G^{\prime}}{B}
$$

Como $B$ tem período finito segue que $G^{\prime}$ tem período finito.

Vamos por fim retirar a hipótese $A$ abeliano, e concluir a passagem (i) $\Longrightarrow$ (ii). Mostraremos também que $G^{\prime}$ é $p$-grupo, e que $G$ tem um subgrupo normal de índice finito $p$-abeliano.

Teorema 4.12. (i) $\Longrightarrow$ (ii).

\section{DEMONSTRAÇÃO:}

Assumindo que $U(F[G])$ satisfaz uma identidade de grupo, por 3.1 temos que $F[G]$ satisfaz uma identidade polinomial. Pelo lema $3.16 G$ tem um subgrupo $A, p$-abeliano, normal de índice finito.

Além disso, pelo lema 3.21 , segue que $G^{\prime}$ é um p-grupo. Resta mostrar, portanto, que $G^{\prime}$ tem período limitado.

Seja $G_{1}=\frac{G}{A^{\prime}}$ e $A_{1}=\frac{A}{A^{\prime}}$. ( $A^{\prime} \triangleleft G$ porque $A^{\prime}$ é subgrupo característico de $\left.A\right)$. 
$A_{1}$ é subgrupo abeliano normal de $G_{1}$, e $\left[G_{1}: A_{1}\right]=[G: A]<\infty$. Além disso pelo lema 4.2, se $U(F[G])$ satisfaz uma identidade de grupo, então $U\left(F\left[G_{1}\right]\right)$ também irá satisfazer.

Agora usando o lema 4.11 segue que $G_{1}^{\prime}$ tem periodo finito. Assim, observando que

$$
G_{1}^{\prime}=\left(\frac{G}{A^{\prime}}\right)^{\prime} \cong \frac{G^{\prime}}{A^{\prime}}
$$

e que $A^{\prime}$ é finito, concluimos que

$$
\operatorname{per}\left(G^{\prime}\right) \leq\left|A^{\prime}\right| \cdot \operatorname{per}\left(G_{1}^{\prime}\right)<\infty
$$

Logo $G^{\prime}$ tem período finito, e assim concluimos a demonstração que (i) $\Longrightarrow$ (ii)

\subsection{A implicação (ii) $\Longrightarrow$ (iii).}

Vamos inicialmente demonstrar o seguinte lema:

Lema 4.13. Seja $R$ uma $F$-álgebra e seja I um ideal de $R$ que é nil de grau limitado $\left(\leq p^{k}\right)$. Se $U\left(\frac{R}{I}\right)$ satisfaz $(x, y)^{p^{j}}=1$, então $U(R)$ satisfaz $(x, y)^{p^{j+k}}=1$.

Demonstração:

Seja

$$
\begin{aligned}
& \varphi: R \longrightarrow \quad \frac{R}{I} \\
& a \longmapsto a \cdot I
\end{aligned}
$$

$\varphi$ induz um homomorfismo sobrejetor de grupos

$$
\varphi: U(R) \longrightarrow U\left(\frac{R}{I}\right) .
$$

Se $x, y \in U(R)$, então,

$$
(\bar{x}, \bar{y})^{p^{j}}=1,(\bar{x}=\varphi(x), \bar{y}=\varphi(y)) .
$$

Logo $(x, y)^{p^{j}}-1 \in I$. Como $I$ é nil de expoente limitado existe $p^{k}$, tal que, para todo $i \in I i^{p^{k}}=0$. Assim

$$
\left((x, y)^{p^{j}}-1\right)^{p^{k}}=(x, y)^{p^{j+k}}-1=0,
$$


e portanto,

$$
(x, y)^{p^{j+k}}=1
$$

como queriamos demonstrar.

Seja $M_{n}(R)$ o anel de matrizes $n \times n$ sobre um anel $R$. Denotaremos por $\left[\alpha_{i, j}\right]$ a matriz pertencente a $M_{n}(R)$, que tem para cada $i$ e $j$ o elemento $\alpha_{i, j} \in R$ na $i$-ésima linha e $j$-ésima coluna.

Lema 4.14. Seja $A$ um subgrupo normal abeliano de $G$ de indice finito $n$, e seja $I$ um ideal de $F[A]$ nil de grau limitado menor ou igual a $p^{k}$ G-invariante (ie $g g^{-1}=$ I para todo $g \in G$ ). Então $I \cdot F[G]$ é um ideal de $F[G]$ que é nil de grau limitado menor ou igual a $n p^{k}$.

\section{DEMONSTRAÇÃO:}

Seja $\left\{g_{1}, \ldots, g_{n}\right\}$ um conjunto completo de representantes para $A$ em $G$, e seja $\varphi$ o homomorfismo:

$$
\begin{aligned}
\varphi: F[G] & \longrightarrow M_{n}(F[A]) \\
\alpha & \longrightarrow\left[\alpha_{i, j}\right]
\end{aligned}
$$

onde $\alpha_{i, j}$ é definida pela fórmula :

$$
g_{i} \cdot \alpha=\sum_{j=1}^{n} \alpha_{i, j} \cdot g_{j}
$$

$\varphi$ está bem definida, visto que a representação de $g_{i} \alpha$ através das classes laterais é única. Vamos mostrar que $\varphi$ é homomorfismo:

É óbvio que vale $\varphi(x+y)=\varphi(x)+\varphi(y)$, vamos mostrar que

$$
\varphi(x y)=\varphi(x) \cdot \varphi(y)
$$

$\operatorname{Sejam} \varphi(x)=\left[\alpha_{i, j}\right]$, e $\varphi(y)=\left[\beta_{i, j}\right]$.

Temos

$$
\begin{gathered}
g_{i} x y=\left(\sum_{k=1}^{n} \alpha_{i, k} g_{k}\right) y= \\
\sum_{k=1}^{n} \sum_{j=1}^{n} \alpha_{i, k} \beta_{k, j} g_{j}=\sum_{j=1}^{n}\left(\sum_{k=1}^{n} \alpha_{i, k} \beta_{k, j}\right) g_{j} .
\end{gathered}
$$


Pela definição de $\varphi(x y)$, concluimos que

$$
\begin{gathered}
\varphi(x y)=\left[\sum_{k=1}^{n} \alpha_{i . k} \cdot \beta_{k, j}\right]=\left[\alpha_{i, j}\right] \cdot\left[\beta_{i, j}\right]= \\
\varphi(x) \cdot \varphi(y) .
\end{gathered}
$$

Além disso se $\varphi(\alpha)=0, \alpha_{i, j}=0$ para cada $i$, e $j$. Logo para todo $i$

$$
g_{i} \alpha=\sum_{j=1}^{n} \alpha_{i, j} g_{j}=0
$$

O que resulta em $\alpha=0$ visto que $g_{i}$ é inversível em $F[G]$. Logo $\varphi$ é injetora.

Certamente $I \cdot F[G]$ é ideal de $F[G]$. Mostraremos agora que $I \cdot F[G]$ é nil de grau limitado menor ou igual a $n p^{k}$. Para tanto demonstraremos que $\varphi(I \cdot F[G]) \leq M_{n}(I)$, e que $M_{n}(I)$ é nil de grau limitado. Do fato que $\varphi$ é injetora segue que $I \cdot F[G]$ é nil de grau limitado.

Vamos mostrar que $\varphi(I \cdot F[G]) \leq M_{n}(I)$. Como $I$ é $G$-invariante, para todo $\alpha \in I \cdot F[G]$, teremos $g_{i} \alpha g_{j}^{-1} \in I \cdot F[\bar{G}]$.

Pelo lema 1.18 , temos que $\alpha_{i, j}=\pi_{A}\left(g_{i} \alpha g_{j}^{-1}\right)$, onde $\pi_{A}$ é a função :

$$
\begin{aligned}
& \pi_{A}: F[G] \longrightarrow F[A] \\
& \alpha=\sum_{g \in G} \alpha_{g} g \longmapsto \\
& \sum_{a \in A} \alpha_{a} a
\end{aligned}
$$

Como $I$ é $G$-invariante e $A \triangleleft G$ temos pelo lema 1.20 que

$$
I=\pi_{A}(I \cdot F[G])
$$

e portanto $\alpha_{i, j} \in I$, para todo $i$, e para todo $j$, logo

$$
\varphi(I \cdot F[G]) \subseteq M_{n}(I)
$$

Vamos provar que $M_{n}(I)$ é nil de grau limitado menor ou igual a $n p^{k}$.

Seja $\sigma \in M_{n}(I)$. Como $I \leq F[A] I$ é comutativo. Seja $p_{\sigma}(x)$ o polinômio característico da matriz $\sigma$

$$
p_{\sigma}(x)=x^{n}-\gamma_{n-1} x^{n-1}-\cdots-\gamma_{0} \text {. }
$$


com $\gamma_{i}$ pertencentes a $I$. Usando o teorema de Cayley-Hamilton (ver teorema 1.15),temos :

$$
\sigma^{n}=\gamma_{0}+\gamma_{1} \cdot \sigma+\cdots+\gamma_{n-1} \cdot \sigma^{n-1}
$$

Elevando os dois lados a $p^{k}$, observando que a característica de $F$ é $p$ e, que $I$ é comutativo temos :

$$
\left(\sigma^{n}\right)^{p^{k}}=\gamma_{0}^{p^{k}}+\gamma_{1}^{p^{k}} \cdot \sigma^{p^{k}}+\cdots+\gamma_{n-1}^{p^{k}} \cdot \sigma^{(n-1) p^{k}}=0,
$$

visto que $I$ é nil de grau limitado, igual a $p^{k}$. Assim concluimos nossa demonstração.

Vamos provar agora (ii) $\Longrightarrow($ iii):

Teorema 4.15. (ii) $\Longrightarrow$ (iii).

DEMONSTRAÇÃO:

Vamos dividir a demonstração em etapas:

1ㅡ etapa. Se existe $A$ central em $G$ tal que $[G: A]<\infty$ e $G^{\prime}$ é p-grupo então $U(F[G])$ satisfaz uma identidade de grupo do tipo $(u, v)^{p^{k}}=1$.

De fato: Se $A$ é central e $[G: A]<\infty$ temos pelo lema 1.3 que $G^{\prime}$ é finito.

Seja

$$
\pi: F[G] \longrightarrow F\left[\frac{G}{G^{\prime}}\right]
$$

o epimorfismo canônico, Pela proposição 1.23, do fato de $G^{\prime}$ ser um $p$-grupo finito, vem que $\operatorname{ker}(\pi)$ é nilpotente.

Mas

$$
F\left[\frac{G}{G^{\prime}}\right]=\frac{F[G]}{\operatorname{ker}(\pi)}
$$

e $U\left(F\left[\frac{G}{G^{\prime}}\right]\right)$ satisfaz $(u, v)=u^{-1} v^{-1} u v=1$. Logo pelo lema 4.13, temos que $U(F[G])$ satisfaz $(u, v)^{p^{k}}$ para algum $k$.

$2^{a}$ etapa. Se $G$ tem um subgrupo normal abeliano $A$ de índice finito, e $G^{\prime} e ́$ p-grupo de período limitado, então $U(F[G])$ satisfaz uma identidade de grupo do tipo $(u, v)^{p^{k}}=1$.

De fato, seja $B=(A, G)$. Um elemento $b$ pertencente a $B$ será da forma:

$$
b=\left(a_{1}, g_{1}\right) \cdots\left(a_{n}, g_{n}\right),
$$


com $a_{i} \in A$ e $g_{i} \in G$. Para todo $h \in G$ temos

$$
h^{-1} b h=h^{-1} a_{1}^{-1} h \cdots h^{-1} a_{n} h \cdot h^{-1} g_{n} h,
$$

Como $A \triangleleft G h^{-1} a h \in A$ para todo $a \in A$. Assim, $h^{-1} b h \in B$ e portanto $B \triangleleft G$.

Como $g_{1}^{-1} a g_{1} \in A, B \leq A$ e $B \leq G^{\prime}$. Portanto

$$
B \leq A \cap G^{\prime}
$$

Logo $B$ é um $p$-grupo abeliano de período limitado.

Seja $\pi: F[A] \longrightarrow F\left[\frac{A}{B}\right]$, o epimorfismo canônico.

Vamos mostrar que $\operatorname{ker}(\pi)$ é nil de grau limitado. De fato

$$
\operatorname{ker}(\pi)=\Delta(B) \cdot F[A],
$$

Seja $p^{j}$ o expoente de $B$, e seja $x \in \operatorname{ker}(\pi)$. Então

$$
x=\sum\left(1-b_{i}\right) \alpha_{i}
$$

com $b_{i} \in B$ e $\alpha_{i} \in F[A]$. Portanto

$$
\begin{gathered}
x^{p^{j}}=\left[\sum\left(1-b_{i}\right) \alpha_{i}\right]^{p^{j}}=\sum\left(1-b_{i}\right)^{p^{j}} \alpha_{i}^{p^{j}}= \\
=\sum\left(1-b_{i}^{p^{j}}\right) \alpha^{p^{j}}=0
\end{gathered}
$$

pois

$$
\left(1-b_{i}^{p^{j}}\right)=0
$$

para todo $b_{i} \in B$.

Além disso

$$
\begin{gathered}
g^{-1} \Delta(B) \cdot F[A] g=g^{-1} \Delta(B) g \cdot g^{-1} F[A] g= \\
=\Delta(B) \cdot F[A],
\end{gathered}
$$

visto que $B \triangleleft G$ e $A \triangleleft G$. Logo $\triangle B F[A]$ é ideal $G$-invariante.

Seja

$$
\pi_{2}: F[G] \longrightarrow F\left[\frac{G}{B}\right]
$$

a projeção canônica.

Temos $\operatorname{ker}\left(\pi_{2}\right)=\Delta(B) \cdot F[G]=(\Delta(B) \cdot F[A]) \cdot F[G]$ 
Como $\Delta B \cdot F[A]$ é $G$-invariante e nil de grau limitado, pelo lema $4.14 \Delta B \cdot F[G]$ é um ideal nil de grau limitado de $F[G]$. Por outro lado $\frac{A}{B}$ é um subgrupo de $\frac{G}{B}$ tal que

$$
a^{-1} g^{-1} a g \in B
$$

e portanto $\frac{A}{B}$ é central em $\frac{G}{B}$.

Além disso $\left[\frac{G}{B}: \frac{A}{B}\right]=[G: A]<\infty$.

Assim $\frac{G}{B}$ tem um subgrupo central de índice finito, e $\frac{G^{\prime}}{B}=\left(\frac{G}{B}\right)^{\prime}$ é p-grupo (pois $G^{\prime}$ é $p$-grupo). Pela $1^{\underline{a}}$ etapa, temos que $U\left(F\left[\frac{G}{B}\right]\right)$ satisfaz uma identidade do tipo,

$$
(u, v)^{p^{l}}=1
$$

Agora $F\left[\frac{G}{B}\right]=\frac{F[G]}{\operatorname{ker}\left(\pi_{2}\right)}$ e $\operatorname{ker}\left(\pi_{2}\right)$ é nil de período limitado. Assim, pelo lema 4.13 $F[G]$ satisfaz uma identidade de grupo do tipo $(u, v)^{p^{k}}=1$.

$3 \underline{\underline{a}}$ etapa. Se $G$ é um grupo de torção, que tem um subgrupo normal p-abeliano $A$ de índice finito, e $G^{\prime}$ é um p-grupo de período limitado então $U(F[G])$ satisfaz uma identidade de grupo do tipo $(u, v)^{p^{k}}=1$.

De fato, seja

$$
\pi_{3}: F[G] \longrightarrow F\left[\frac{G}{A^{\prime}}\right],
$$

o epimorfismo canônico. Como $A^{\prime}$ é $p$-grupo finito segue pelo lema 1.23 que $\operatorname{ker}\left(\pi_{3}\right)$ é nilpotente.

Por outro lado $\frac{G}{A^{\prime}}$ tem um subgrupo normal abeliano de índice finito $\frac{A}{A^{\prime}}$, e $\left(\frac{G}{A^{\prime}}\right)^{\prime} \cong \frac{G^{\prime}}{A^{\prime} \cap G^{\prime}}$ é $p$-grupo de período limitado. Pela $2^{\underline{a}}$ etapa podemos concluir que $U\left(F\left[\frac{G}{A^{\prime}}\right]\right)$ satisfaz uma identidade de grupo do tipo $(u, v)^{p^{j}}=1$.

Assim, pelo lema 4.13, temos que $U(F[G])$ satisfaz uma identidade de grupo do tipo

$$
(u, v)^{p^{k}}=1
$$

concluindo assim a demonstração de (ii) $\Longrightarrow$ (iii).

Como (iii) $\Longrightarrow$ (i) é trivial concluimos a demonstração do teorema. 


\section{Bibliografia}

[BRT] Y.Billig, D.Riley, e V.Tasic, Non-matrix varieties and nil generated algebras whose units satisfy a group identity. (preprint)

[DG97] M.A.Dokuchaev, e J.Z.Gonçalves, Semigroup identity on units of integral group rings. Glasgow Math. Journal, 39 (1997) pp 1-6

[GJV94] A.Giambruno, E.Jespers, e A.Valenti, Group identities on units of rings. Arch.Math. 63 (1994) pp 241-243

[GSV97] A.Giambruno, S.K.Sehgal, e A.Valenti, Group algebras whose units satisfy a group identity. Proceedings of The American Mathematical Society. 125, (1997) pp 629-634.

[JZG84] J.Z.Gonçalves, Free groups of units in group rings. Canadian Mathematical Bulletin 27 (1984) pp 309-312.

[GM91] J.Z.Gonçalves, e A.Mandel, Semigroups identities on units of group algebras. Arch.Math. 57 (1991) pp 539-545.

[GP96] J.Z.Gonçalves, D.S.Passman, Construction of free subgroups in the groups of units of modular group algebra. Comunications in Algebra, 24 (13), 4211-4215 (1996)

[HP80] B.Hartley, P.F.Pickel, Free subgroups in the unit groups of integral group rings. Canadian Journal of Mathematics, vol 32, num:6, (1980), pp 1342-1352.

[Lam91] T.Y.Lam, A first course in non-comutative rings. Springer Verlag, New York, 1991.

[KD93] H.Kopka e P.W.Daly, A guide to Latex 2є: document preparation from beginners and advanced users 2nd edition. Addison-Wesley Publishing Company, 1993. 
[Liu] C.H.Liu, Group algebras with units satisfying a group identity. (preprint)

[LS77] R.C.Lyndon, e P.E.Schupp, Combinatorial group theory. Springer Verlag, Berlin, 1977.

[MKS76] W.Magnus, A.Karras, e D.Solitar, Combinatorial group theory: presentations of groups in terms of generators and relations. Dover Publications Inc., New York, 1976.

[Pas71] D.S.Passman, Group rings satisfying a polynomial identity II. Pacific Journal of Mathmatics, 39 (1971) pp 425-438

[Pas77] D.S.Passman, The algebraic struture of group rings. John Willey and Sons, New York 1977.

[Pas97] D.S.Passman, Group algebras whose units satisfy a group identity II. Proceedings of The American Mathematical Society. 125, (1997) pp 657-662.

[Rob95] D.J.S.Robinson, A course in the theory of groups 2nd edition. Springer Verlag, New York, 1995.

[Row80] L.H.Rowen, Polynomial identities in ring theory. Academic Press, LondonNew York San Francisco. 1980.

[Sco87] W.R.Scott, Group theory. Dover Publications Inc., New York, 1987.

[Seh78] S.K.Sehgal, Topics in group rings. Marcel Dekker, New York, 1978.

[Seh93] S.K.Sehgal, Units in integral group rings. Longman, Essex, 1993.

[Tits72] J.Tits, Free subgroups in linear groups. Journal of Algebra, 20, (1972), pp 250-270.

[War81] D.S.Warhurst, Topics in group rings. Thesis Manchester, 1981. 